\title{
Alternatively activated macrophages determine repair of the infarcted adult murine heart
}

\author{
Manabu Shiraishi, ${ }^{1,2}$ Yasunori Shintani, ${ }^{1}$ Yusuke Shintani, ${ }^{1}$ Hidekazu Ishida, ${ }^{1}$ Rie Saba, ${ }^{1}$ Atsushi Yamaguchi, ${ }^{2}$ Hideo Adachi, ${ }^{2}$ \\ Kenta Yashiro, ${ }^{1}$ and Ken Suzuki' \\ 'William Harvey Research Institute, Barts and The London School of Medicine and Dentistry, Queen Mary University of London, London, United Kingdom. ²Department of Cardiovascular Surgery, \\ Saitama Medical Center, Jichi Medical University, Saitama, Japan.
}

\begin{abstract}
Alternatively activated (also known as M2) macrophages are involved in the repair of various types of organs. However, the contribution of $\mathrm{M} 2$ macrophages to cardiac repair after myocardial infarction (MI) remains to be fully characterized. Here, we identified $\mathrm{CD} 206+\mathrm{F} 4 / 80+C D 11 b^{+} \mathrm{M} 2$-like macrophages in the murine heart and demonstrated that this cell population predominantly increases in the infarct area and exhibits strengthened reparative abilities after MI. We evaluated mice lacking the kinase TRIB1 ( Trib1 $^{-1-}$ ), which exhibit a selective depletion of M2 macrophages after MI. Compared with control animals, Trib1 $^{-1-}$ mice had a catastrophic prognosis, with frequent cardiac rupture, as the result of markedly reduced collagen fibril formation in the infarct area due to impaired fibroblast activation. The decreased tissue repair observed in Trib1 ${ }^{-1-}$ mice was entirely rescued by an external supply of M2-like macrophages. Furthermore, IL-1 $\alpha$ and osteopontin were suggested to be mediators of M2-like macrophage-induced fibroblast activation. In addition, IL-4 administration achieved a targeted increase in the number of M2-like macrophages and enhanced the post-MI prognosis of WT mice, corresponding with amplified fibroblast activation and formation of more supportive fibrous tissues in the infarcts. Together, these data demonstrate that M2-like macrophages critically determine the repair of infarcted adult murine heart by regulating fibroblast activation and suggest that IL-4 is a potential biological drug for treating MI.
\end{abstract}

\section{Introduction}

The formation of connective tissue is an essential process in the healing and repair of almost every tissue and organ, and this process is of particular importance in the case of myocardial repair $(1,2)$. Because of its insufficient regenerative ability $(3)$, the adult mammalian heart must permanently compensate for the post-MI loss of cardiomyocytes by producing fibrotic tissues. These connective tissues must be firm and extensive enough to maintain the rigidity and performance of the failing heart in conditions of high mechanical stress. Without this, the fragile ventricular wall will undergo sudden rupture in the worst-case scenario. Even if cardiac rupture does not occur, the insufficient tissue repair will make the damaged heart more vulnerable to adverse ventricular remodeling, and this condition will progressively advance to endstage heart failure $(1,2)$.

Alternatively activated (or M2) macrophages play a role in the repair and/or regeneration of various types of organs, and the features of these cells are distinct in different organs and environmental conditions (4-6). Recent studies have implicated the involvement of $\mathrm{M} 2$ macrophages in myocardial repair by depleting macrophages with chemicals (e.g., clodronate liposome) or knocking out genes that are relevant to myeloid cell differentiation (7-13). However, these depletion methods have limited specificity for M2 macrophages. These methods deplete whole macrophages, including not only M2 macrophages but also proinflammatory (M1) macrophages

Conflict of interest: The authors have declared that no conflict of interest exists. Submitted: December 1, 2015; Accepted: March 10, 2016.

Reference information: J Clin Invest. 2016;126(6):2151-2166. doi:10.1172/JCI85782. and other cells (14). Therefore, these methods are useful for investigating the overall contribution of whole macrophage subsets; however, these methods have a limited capability in dissecting the precise role of a certain subset of macrophages (i.e., M2 macrophages). In addition, "rescue" (compensation of depleted M2 macrophages) experiments have not been applied in these studies. Similarly, it was not possible for these models to identify the cellular and molecular mechanisms by which a particular subset of macrophages (i.e., M2 macrophages) induce myocardial repair. Therefore, it is important to accumulate more convincing evidence to determine the precise role of M2 macrophages after MI using a more appropriate model, which will also help to dissect the underlying mechanism (15). To this end, we used mice with a deletion of the Trib1 gene (a member of the $\mathrm{Ca}^{2+} /$ calmodulin-dependent protein kinase [CAMK] Ser/ Thr protein kinase family), which have been shown to possess an impaired ability to form M2 macrophages in the spleen, liver, lung, and adipose tissue, whereas Ly-6C ${ }^{\text {hi }}$ inflammatory monocytes or macrophages, lymphocytes, neutrophils, and dendritic cells (DCs) are unaffected (16). We hypothesized that this mouse would be able to offer a more specific depletion of M2 macrophages in the heart and thus enable us to elucidate the precise contributions of M2 macrophages to myocardial repair after MI.

Although recent progress in diagnosis and treatment, including percutaneous coronary interventions, has significantly improved the early survival of patients who suffer an MI, this disease remains one of the major causes of human death and disability (17). Therefore, the development of new, more effective treatments is of urgent importance. One promising approach to achieve this goal may be to increase the number of cardiac M2 

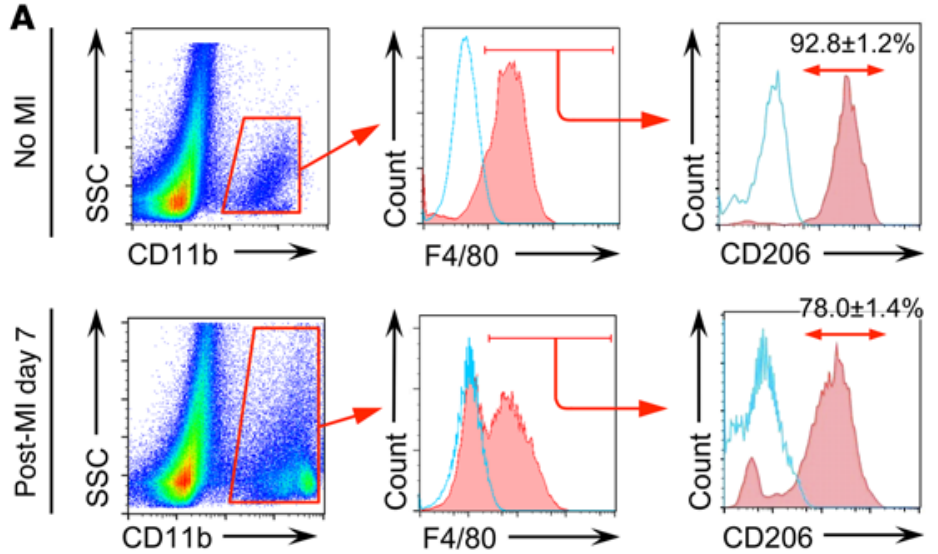

B

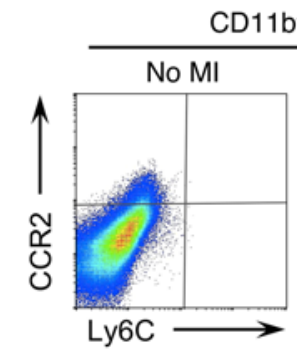

CD11b+CD206 ${ }^{+}$cells
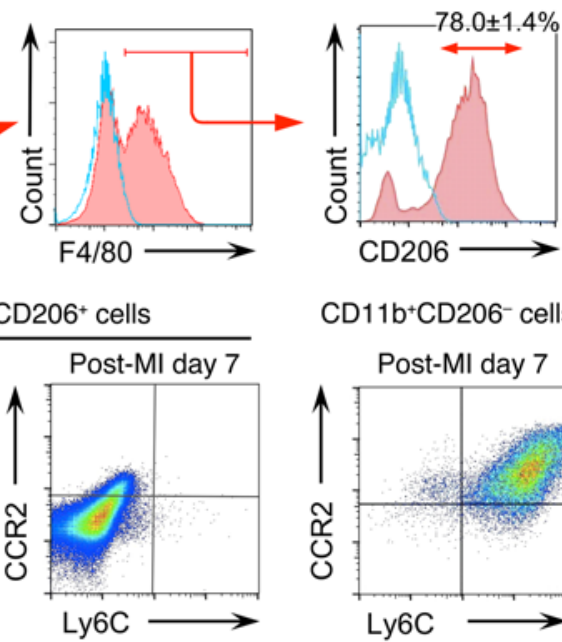

CD11b+CD206- cells

Post-MI day 7

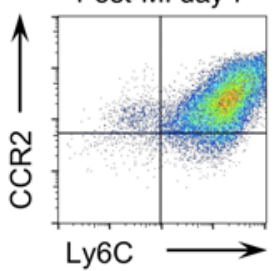

C
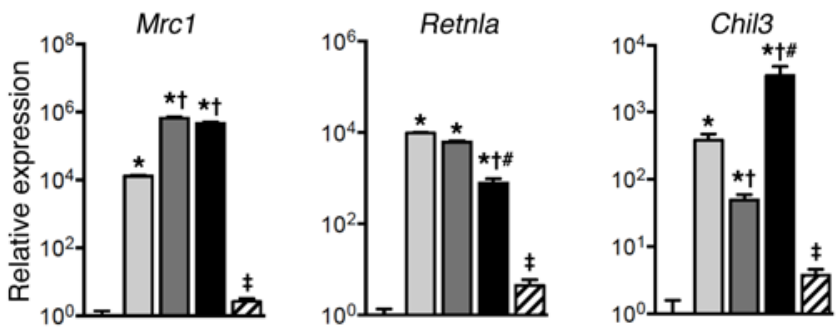

Fibro

$\square$ M2 (Peritoneal) $\square$ M2 (no MI)

M2 (MI) $\mathbb{Z}$ M1 (MI) macrophages $(18,19)$; however, the methods used to enhance cardiac M2 macrophages in previous studies are clinically unfeasible, unsuccessful, or under development (20-22). IL-4 is a major Th2 cytokine that drives the differentiation of monocytes and macrophages into M2 phenotypes and/or increases their proliferation in situ, as observed in tissues other than the heart (23-26). Thus, we investigated the potential for IL-4 administration to increase M2 macrophages in the heart after MI, improve the repair of the damaged myocardium, and enhance the maintenance of the function and structure of the infarcted heart.

\section{Results}

M2-like macrophages were present in the adult murine heart and were predominantly increased in the infarct area after MI, exhibiting strengthened reparative abilities. We confirmed that the left ventricular (LV) myocardium of adult mice contained $\mathrm{CD} 11 \mathrm{~b}^{+} \mathrm{F} 4 / 80^{+}$ macrophages, more than $90 \%$ of which were positive for CD206 (Figure 1A). On day 7 after MI that was induced by coronary artery ligation, the ratio of $\mathrm{CD} 206^{+} \mathrm{CD} 11 \mathrm{~b}^{+} \mathrm{F} 4 / 80^{+}$macrophages was reduced, with an increase in the CD206- subset. Conversely, the majority of $\mathrm{CD}^{2} \mathrm{O6}^{+}$cardiac cells were positive for both F4/80 and CD11b in both normal (no MI) and post-MI hearts (Supplemental Figure 1; supplemental material available online
Figure 1. M2-like macrophages were present in the adult murine heart. (A) Flow cytometric analysis confirmed that $\mathrm{CD}^{206}{ }^{+} \mathrm{F} 4 / 80^{+} \mathrm{CD} 1 \mathrm{~b}^{+} \mathrm{M} 2$-like macrophages were present in normal, no-MI hearts and in day-7 post-MI hearts of adult C57BL/6 mice. More than $90 \%$ of $\mathrm{F} 4 / 80^{+} \mathrm{CD} 11 \mathrm{~b}^{+}$macrophages in normal hearts were positive for CD206, whereas the percentage of $C D 206^{+}$cells in $\mathrm{F} 4 / 80^{+} \mathrm{CD} 11 \mathrm{~b}^{+}$macrophages was reduced to $78.0 \% \pm 1.4 \%$ at day 7 after MI ( $n=6$ in each group; $P<0.01,2$-tailed, unpaired Student's $t$ test). Light blue lines indicate the IgG control data. (B) Most $(92.7 \%$ $\pm 1.4 \%$ and $90.0 \% \pm 2.6 \%$ in the no-MI hearts and day-7 post-MI hearts) of the CD11b+CD206 ${ }^{+}$cells were CCR2- and $\mathrm{Ly}^{-} \mathrm{CC}^{-}$, while $97.1 \%$ $\pm 1.0 \%$ of $\mathrm{CD} 11 \mathrm{~b}^{+} \mathrm{CD} 206^{-}$cells were CCR2 ${ }^{+}$and $\mathrm{LyC6}^{+}$. Representative results of 6 different studies are presented. (C) CD206 $F 4 / 80^{+} C^{2} 11 b^{+}$ M2-like macrophages were collected by FACS from the peritoneal cavity of normal mice [M2 (Peritoneal)], from intact, no-MI hearts [M2 (no MI)], and from day-7 post-MI hearts [M2 (MI)] and subjected to real-time reverse transcription PCR (RT-PCR) analysis. CD11b+CD206- M1-like macrophages were also collected from day-7 post-MI hearts [M1 (MI)] and analyzed. Relative expression to that of cardiac fibroblasts (Fibro) is presented. M2-like macrophages from all 3 sources expressed the M2 macrophage markers Mrc1 (CD206), Retnla (Fizz1), and Chil3 (Ym1). $n=6$ different mice in each group. ${ }^{*} P<0.05$ versus both Fibro and $\mathrm{M} 1(\mathrm{MI}) ;{ }^{\dagger} P<0.05$ versus $\mathrm{M} 2$ (Peritoneal); ${ }^{\#} P<0.05$ versus M2 (no MI); ${ }^{\ddagger} P<0.05$ versus Fibro; 1-way ANOVA. with this article; doi:10.1172/JCI85782DS1). CD11b ${ }^{+}$CD206 ${ }^{+}$cardiac cells were primarily negative for CCR2 and Ly6C, whereas

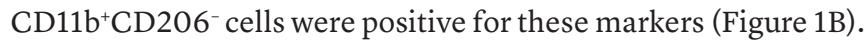
In addition, these $\mathrm{CD} 206^{+} \mathrm{F} 4 / 80^{+} \mathrm{CD} 1 \mathrm{~b}^{+}$cells expressed high levels of other M2 macrophage markers including Chil3 (Ym1) and Retnla (Fizz1) $(4-6,27)$, both before and after MI, compared with what was detected in primary cardiac fibroblasts and $\mathrm{CD} 11 \mathrm{~b}^{+} \mathrm{CD} 206^{-}$cardiac cells (Figure 1C).

Consistent with these findings, a sizable number of CD206 ${ }^{+}$ cells were detected by IHC in the normal heart (Figure 2A). Approximately $96.9 \% \pm 2.0 \%$ of these cells were positive for CD 68 , whereas $92.8 \% \pm 3.0 \%$ of the $\mathrm{CD}^{2} 8^{+}$macrophages were CD206 ${ }^{+}$. After MI, CD206 ${ }^{+}$cells exhibited dynamic spatiotemporal changes in their distribution. These cells were predominantly increased in the infarct area (and in the border area to a lesser degree), with a peak on day 7 (Figure 2, B and C). In contrast, CD11 $\mathrm{c}^{+}$cells were rarely observed in the intact (no-MI) heart but increased by day 3 after MI in the infarct and border areas (Figure $2 \mathrm{C}$ and Supplemental Figure 2B). Although it is not entirely specific (i.e., inclusion of DCs), CD11c is a frequently used cell-surface marker for M1-like inflammatory macrophages and monocytes (28-30). Our data supported the utility of CD11c by demonstrating that most of the CD11 $\mathrm{c}^{+}$cells from the day-7 post-MI heart were 
A

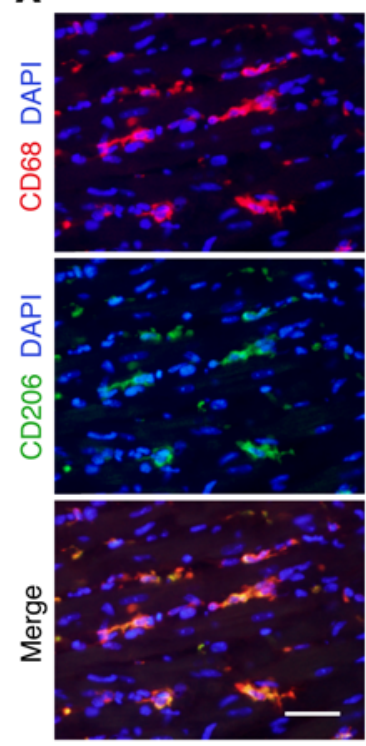

B

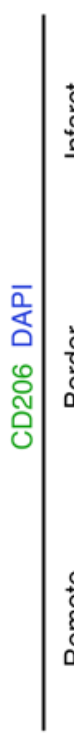

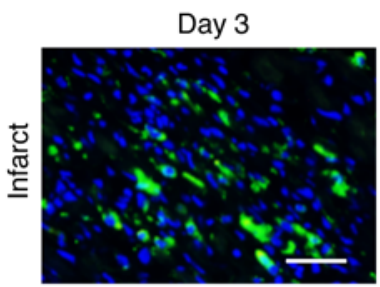
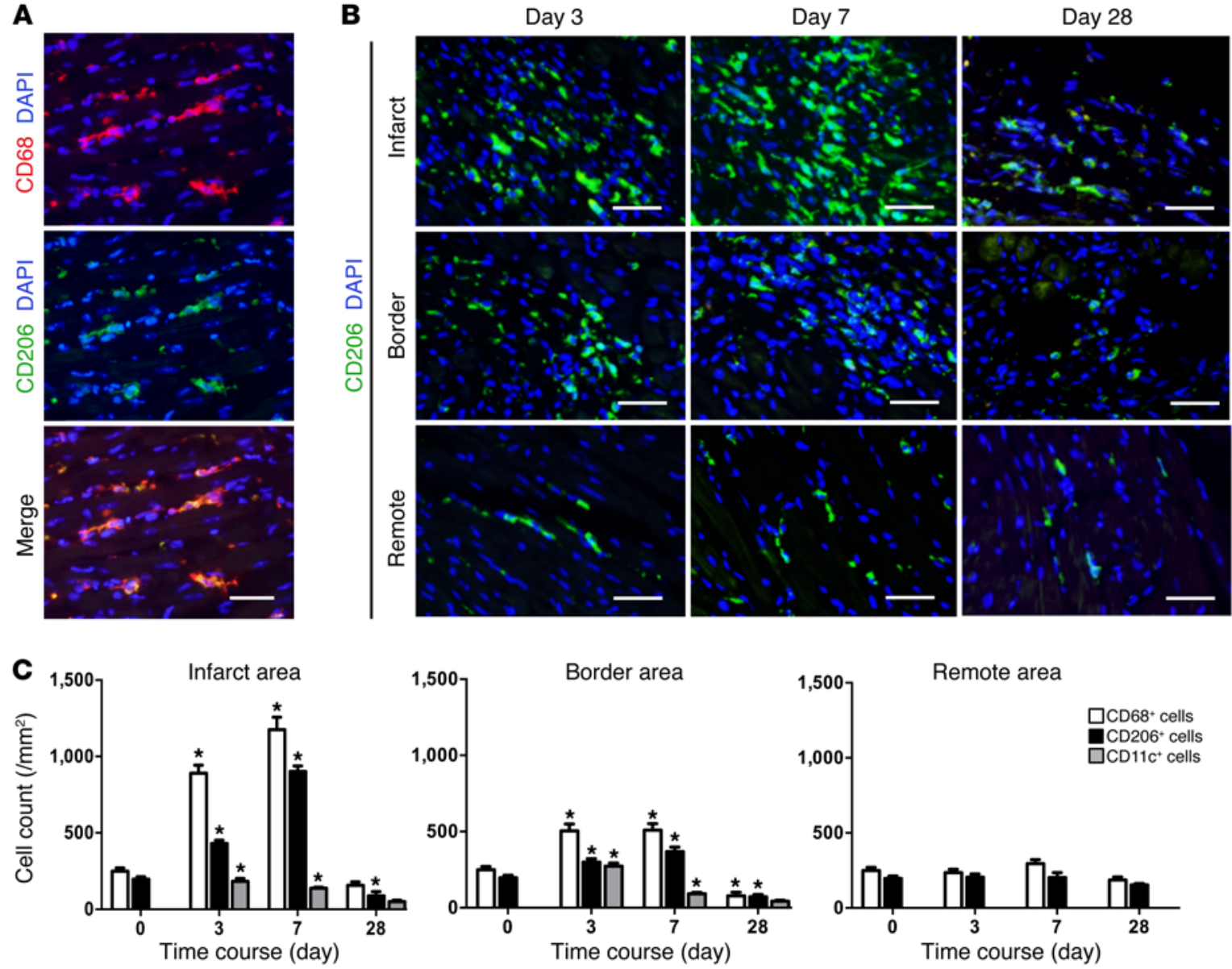

Figure 2. Cardiac M2-like macrophages underwent post-MI area-specific changes. (A) IHC confirmed the presence of CD2O6+ cells in the myocardial interstitium of normal mouse hearts, almost all of which (96.9\% $\pm 2.0 \% ; n=6$ different hearts) were also positive for CD68. Conversely, the majority $(92.8 \% \pm 3.0 \% ; n=6$ different hearts) of CD68+ cells were CD206+. Nuclei were stained with DAPI. Scale bar: $50 \mu \mathrm{m}$. (B) Immunofluorescence revealed that the number of $\mathrm{CD} \mathrm{OG}^{+} \mathrm{M} 2$-like macrophages was primarily increased in the infarct area (and in the border area to a lesser extent), with a post-MI peak on day 7. No change in the number of M2-like macrophages in the remote area was noted. Scale bars: $50 \mu \mathrm{m}$. (C) The post-MI chronological numbers of CD68+, $\mathrm{CD}^{2} \mathrm{C}^{+}$, and $\mathrm{CD} 11 \mathrm{C}^{+}$cells in each infarct, border, or remote area were counted in the immunohistochemical samples described above. See Supplemental Figure 2 for representative images of immunostaining for CD68 and CD11c. $n=6$ different hearts. ${ }^{*} P<0.05$ versus day 0 (no MI, normal heart) in each cell type, repeated-measures ANOVA.

$\mathrm{CCR}^{+}{ }^{+} \mathrm{Ly}_{6 \mathrm{C}}{ }^{+} \mathrm{CD} 206^{-}$(Supplemental Figure 3 ) and that CD11c was

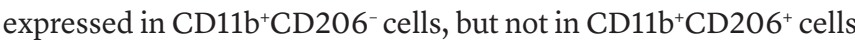
(Supplemental Figure 4). Additionally, it is known that the post-MI frequency of cardiac DCs is much lower (fewer than 1 in 20 cells) that that of cardiac macrophages throughout (31), suggesting that contamination of $\mathrm{CD} 11 \mathrm{c}^{+}$cardiac cells by DCs is insignificant, if it occurs at all. Furthermore, the number of $\mathrm{CD}^{+} 8^{+}$cells (pan-macrophages) was generally equal to the sum of CD206 ${ }^{+}$cells and CD11 $\mathrm{c}^{+}$cells in each area at each time point after MI (Figure 2C and Supplemental Figure 2).

Microarray analysis revealed that $\mathrm{CD} 206^{+} \mathrm{F} 4 / 80^{+} \mathrm{CD}_{11 \mathrm{~b}^{+}}$ M2-like macrophages collected from day-7 post-MI hearts had a different molecular signature than did those from intact hearts, although both were clustered in a group that was distinct from peritoneal M2 macrophages (Figure 3A; full data are available in the Gene Expression Omnibus [GEO] database; GSE69879). A range of antiinflammatory and repair-associated genes, including Il1O, Il1rn, Vegfa, Il1a, and Spp1 (osteopontin) (32-38), were upregulated in M2-like macrophages from MI hearts compared with intact hearts (Figure 3, B and C). The expression levels of these repairassociated genes in the $\mathrm{CD} 206^{+}$cardiac M2-like macrophages were

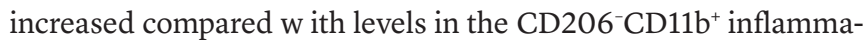
tory (M1-like) macrophages and monocytes from post-MI hearts.

These results suggest that M2-like macrophages increase in number and also exhibit increases in post-MI antiinflammation and tissue repair activity at the appropriate location and time at which inflammation resolution and tissue healing are necessary. These findings imply a potential role for these cells in the repair of infarcted myocardium.

Trib1 $1^{-1-}$ mice showed a depletion of the post-MI augmentation of M2-like macrophages, without altering M1-like macrophages. We next aimed to clarify the reparative role of post-MI CD206 ${ }^{+}$M2-like macrophages using $\mathrm{Trib1}^{-1-}$ mice, which are known to have an impaired ability to form M2-like macrophages in the spleen, liver, lung, and adipose tissue, while their ability to form other inflammatory cells remains intact (16). We first confirmed that the heart function and structure, extracellular collagen deposition, occurrence of cardiac fibroblasts, and capillary density of the intact, 

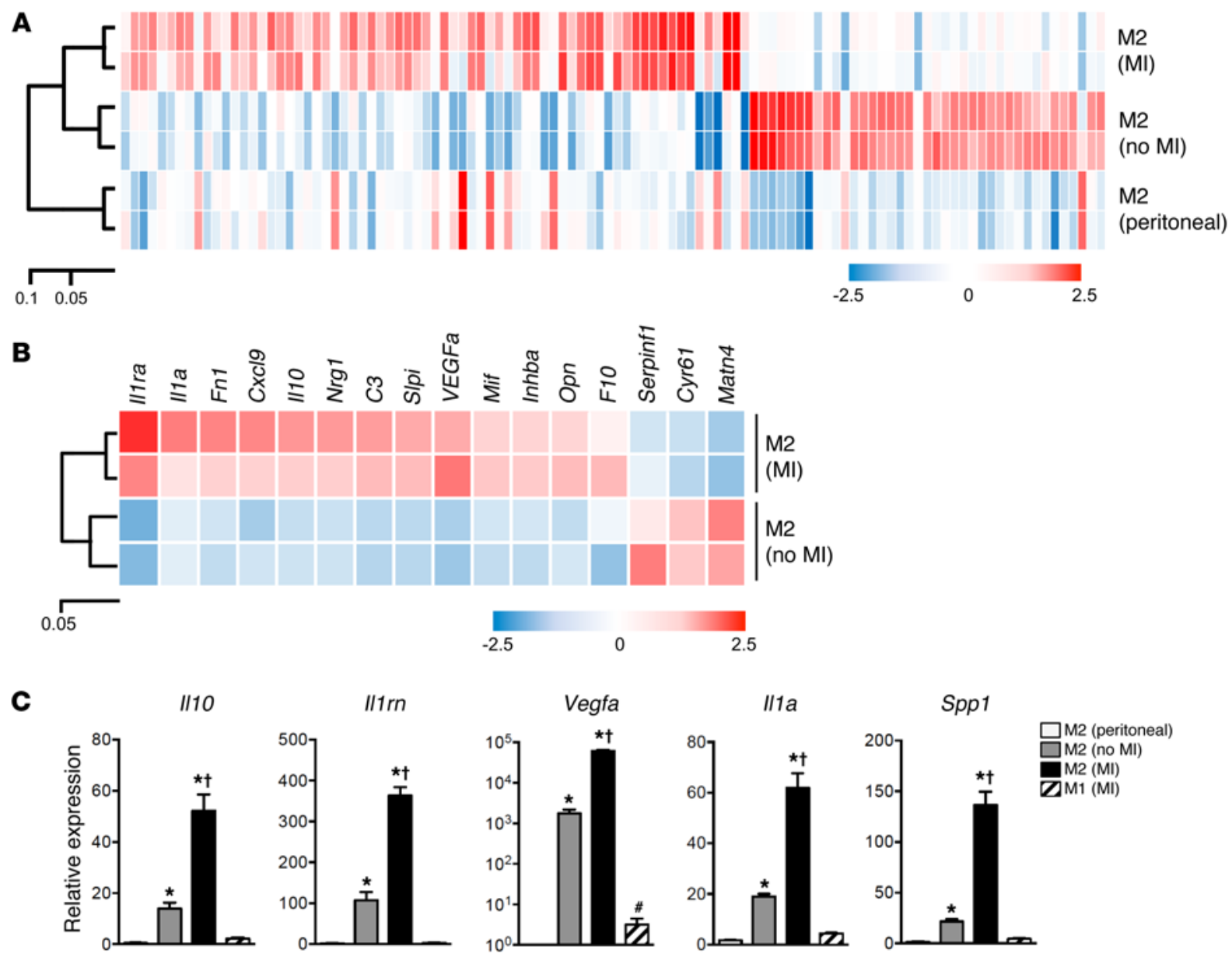

Figure 3. Cardiac M2-like macrophages strengthened their post-MI reparative ability. (A) CD206+F4/80+CD11b+ M2-like macrophages were collected by FACS from intact hearts [M2 (no MI)], day-7 post-MI hearts [M2 (MI)], and from the peritoneal cavity of intact mice [(M2 (Peritoneal)] and subjected to microarray analysis. Macrophages from different origins showed distinct molecular signatures. M2 (MI) had a different expression profile from that of M2 (no MI), although both were clustered distinctly from the M2 (Peritoneal) profile. (B) Heatmap revealed that among the genes encoding secreted proteins, 13 genes were upregulated and 3 were downregulated in M2 (MI) compared with M2 (no MI) macrophages. The upregulated genes included antiinflammatory and repair-associated genes. (C) Quantitative RT-PCR ( $($ RT-PCR) analysis confirmed the post-Ml upregulation of these repair-associated genes in $\mathrm{CD}_{206}{ }^{+} \mathrm{F} 4 / 80^{+} \mathrm{CD}_{11 \mathrm{~b}^{+}}$cardiac M2-like macrophages [M2 (MI)]. CD11 ${ }^{+} \mathrm{CD} 206^{-}$M1-like macrophages from day-7 post-MI hearts [M1 (MI)] expressed these genes at lower levels. Expression levels relative to those of M2 (Peritoneal) are presented. $n=6$ in each group. ${ }^{*} P<0.05$ versus both M2 (Peritoneal) and M1 (MI); ${ }^{\dagger} P<0.05$ versus M2 (no MI); ${ }^{\#} P<0.05$ versus M2 (Peritoneal); 1 -way ANOVA.

no-MI hearts of Trib1 $^{-1-}$ mice were comparable to those of their WT littermates (Supplemental Table 1 and Supplemental Figure 5). Although the presence of M2-like macrophages in intact hearts was similar in Trib1 $1^{-1}$ and WT mice, the post-MI augmentation of M2-like macrophages in damaged areas was obviously eliminated in Trib1 ${ }^{--}$mice (Figure 4, A-C). The $\mathrm{Ki}^{-} 7^{+}$ratio of $\mathrm{CD} 206^{+}$ M2-like macrophages was similar in normal, no-MI hearts from Trib1 $^{-/}$and WT mice, whereas the percentage of Ki67 cells after MI was increased only in WT mice (Supplemental Figure 6). The presence of $\mathrm{CD}_{11} \mathrm{c}^{+}$cells (mostly proinflammatory macrophages and monocytes) was identical in these mice, both before and after MI (Supplemental Figure 7). We attempted to recover the post-MI deficiency in M2-like macrophages in Trib1/- mice by transplanting BM-derived macrophages (BMDMs) collected from WT mice. Consistent with previous findings in the lung (39), we confirmed that BMDM transplantation achieved a frequency of $\mathrm{CD} 206^{+}$ macrophages in the infarct area similar to that detected in their day-7 post-MI WT littermates (Figure 4D). In contrast, there was no such accumulation of BMDM-derived CD206 ${ }^{+}$macrophages in the remote area. Flow cytometry demonstrated that the transplanted (CM-DiI ${ }^{+}$) BMDMs were CD206 ${ }^{+}(97.3 \%), \mathrm{F} 4 / 80^{+}$ (70.6\%), CCR2- and Ly6C- (Supplemental Figure 8), suggesting that these cells had a phenotype equivalent to that of cardiac M2-like macrophages (refer to Figure 1).

Suppression of M2-like macrophages in Trib1-- mice resulted in a catastrophic post-MI prognosis, with impaired fibroblast-mediated repair of the infarcted myocardium. The abolished accumulation of CD206 ${ }^{+}$M2-like macrophages in the hearts of Trib1 ${ }^{-1}$ mice resulted in a catastrophic post-MI prognosis; the majority of Trib1/mice died during the initial week following MI (Figure 5A). In particular, $\mathrm{Trib}^{-/-}$mice developed cardiac rupture 9-fold more frequently than did their WT littermates (Figure 5B). However, transplantation of WT-derived BMDMs incorporated in Matrigel, which compensated for the deficiency in cardiac M2-like macro- 
A

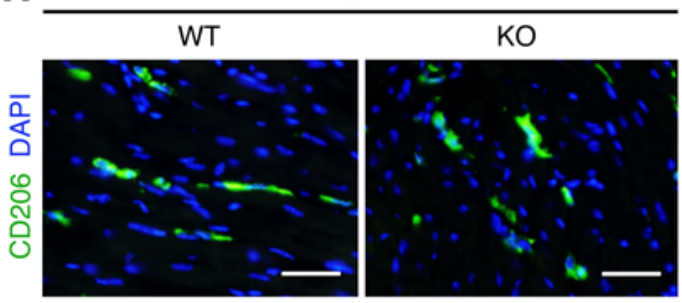

B

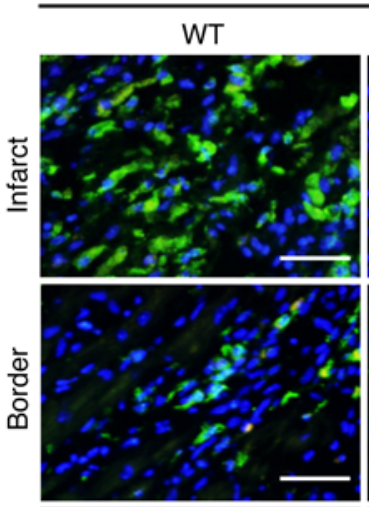

Post-MI day 7

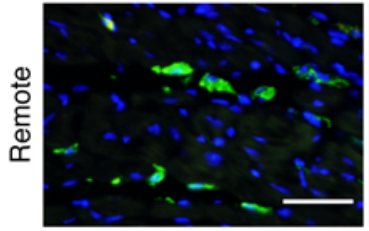

CD206 DAPI
C
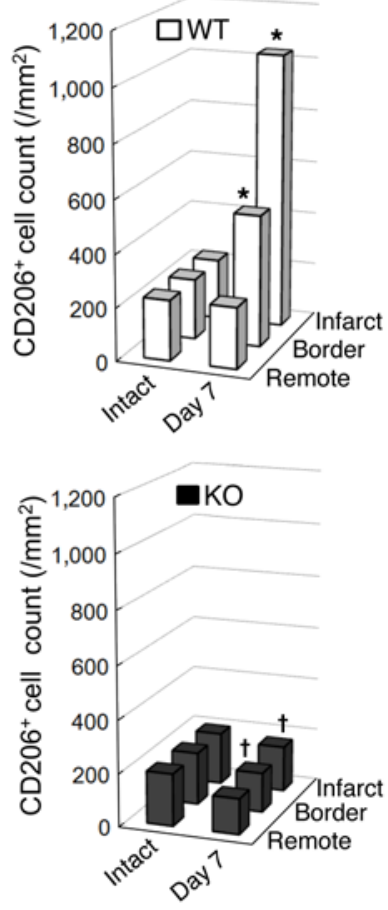

Figure 4. Post-MI increase in M2-like macrophages was abolished in $\mathrm{Trib1}^{-/-}$mice. (A) IHC indicated that the presence of CD206 ${ }^{+}$ $\mathrm{M} 2$-like macrophages in intact, no-Ml hearts in the $\mathrm{KO}$ group ( $\mathrm{Trib}^{1 /-}$ mice) was similar to that seen in the WT littermates group. $n=6$ hearts in each group. Scale bars: $50 \mu \mathrm{m}$. (B) The increased number of CD206 ${ }^{+}$M2-like macrophages on day 7 after $\mathrm{MI}$ in the infarct and border areas of the WT group hearts $(n=6)$ was completely eliminated in the $\mathrm{KO}$ group hearts $(n=5)$. Scale bars: $50 \mu \mathrm{m}$. (C) The numbers of CD206 ${ }^{+}$ cells were serially counted in each area. It was evident that the post-MI increase in $\mathrm{CD}^{2} \mathrm{6}^{+} \mathrm{M} 2$-like macrophage numbers in the damaged myocardium was abolished in rrib1 $^{-/-}$mice. $n=5-6$ for each point in each group. ${ }^{*} P<0.05$ versus intact, no-MI hearts in each group; ${ }^{\dagger} P<0.05$ versus the WT group at the corresponding time and area; repeated-measures ANOVA. (D) On day 7 after BMDM transplantation (WT mice derived and labeled with (M-Dil) into $\mathrm{KO}\left(\right.$ Trib1 $\left.^{-1-}\right)$ mice with MI, the frequency of $\mathrm{CD}_{206}{ }^{\mathrm{M}} \mathrm{M}$-like macrophages in the infarct area was recovered to a degree similar to that in the WT group (see Figure 4, B and C). Most of the $\mathrm{CD}^{206^{+}} \mathrm{M} 2$ macrophages originated from the donor cells (Host, CD206+CM-Dil- cells; Donor, CD206+CM-Dil ${ }^{+}$ cells). Scale bar: $50 \mu \mathrm{m} . n=6$ hearts.

\section{D}

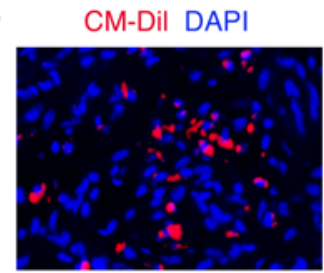

CD206 DAPI

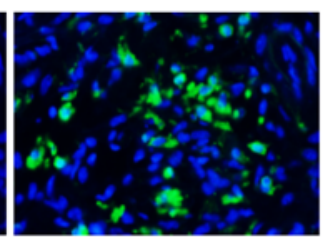

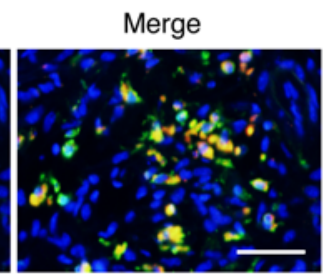

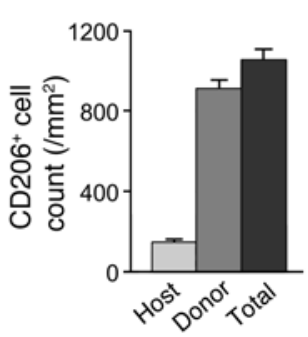

phages (Figure 4D and Supplemental Figure 8), rescued this poor prognosis and frequent cardiac rupture after MI (Figure 5, A and B). Transplantation of Matrigel only (without cells) did not affect the mortality or cardiac rupture rates of Trib1 $1^{-1}$ mice. Echocardiography revealed that post-MI cardiac dysfunction and dilatation were exacerbated in Trib1 ${ }^{-/}$mice compared with what was observed in their WT littermates, whereas this disorder was normalized by BMDM transplantation (Figure 5C and Supplemental Table 1). Histological studies demonstrated that suppression of the post-MI accumulation of $\mathrm{CD} 2 \mathrm{O6}^{+}$M2-like macrophages in Trib1-/ mouse hearts was associated with a repressed formation of connective tissues. Collagen deposition in the infarct area was markedly decreased, with reduced accumulation and activation of cardiac fibroblasts (Figure 6 and Supplemental Figures 9 and 10). Importantly, the impaired fibroblast activation and diminished formation of fibrotic tissues in the infarct area in Trib1 ${ }^{-/}$mice were successfully restored by transplanting BMDMs from WT mice.
These data indicate that M2-like macrophages are essential for post-MI fibroblast-mediated cardiac repair.

IL-4 administration amplified the post-MI augmentation of cardiac M2-like macrophages. The establishment of an effective method to amplify cardiac M2-like macrophages is valuable for the purposes of strengthening the above data to support the biological roles of these cells in myocardial repair as well as developing an innovative treatment for heart failure $(18,19)$. Here, we demonstrated that a pharmacological approach using IL-4 helped to achieve this goal. WT mice received i.p. injections of either a long-acting IL-4 complex (23) or PBS (control) and then underwent the MI-induction surgery. As expected, IL-4 treatment amplified ( $>2$-fold) the post-MI increase in CD206 $6^{+}$macrophages in the damaged myocardium compared with that seen in the control (Figure 7). The percentage of $\mathrm{Ki}^{6} 7^{+}$-expressing CD206 ${ }^{+}$ cardiac M2-like macrophages before MI was increased with IL-4 treatment (Supplemental Figure 11). Furthermore, although the 

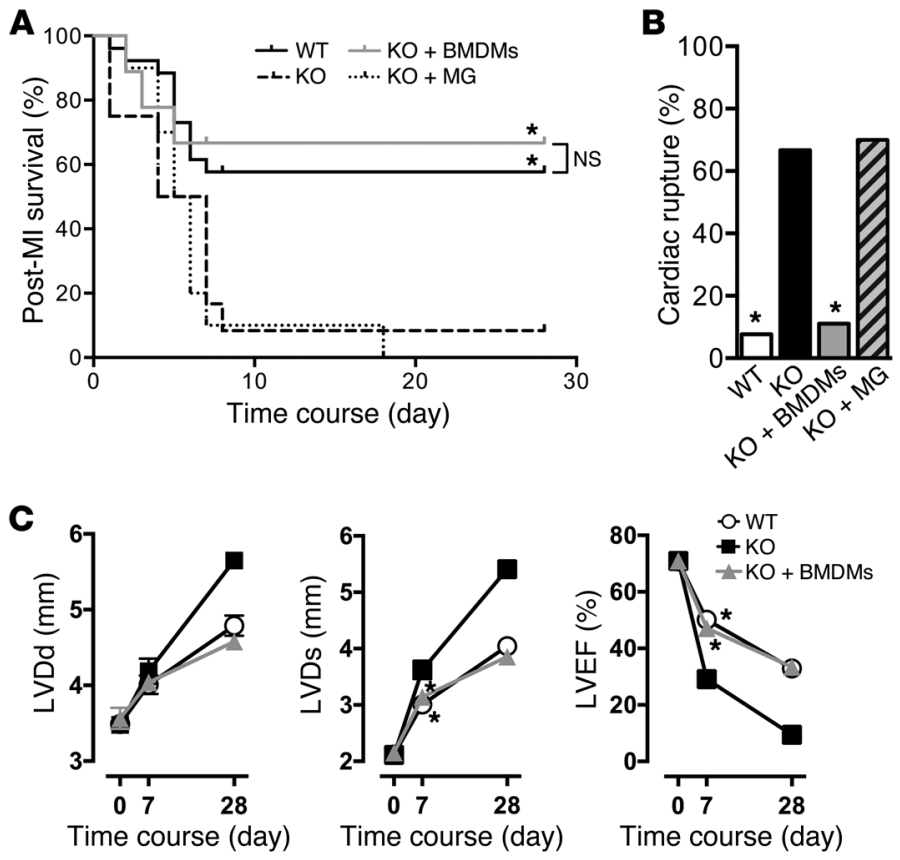

proliferation of $\mathrm{CD}^{2} 26^{+}$cells in the control group was increased after $\mathrm{MI}$, this post-MI increase in the proliferation of CD206 $6^{+}$cardiac cells was more extensive following IL-4 treatment.

$I L-4$ treatment improved post-MI cardiac performance by strengthening the fibroblast-mediated formation of connective tissues in the infarct area. IL-4-mediated amplification of the post-MI increase in M2-like macrophages was associated with improved survival and prevented cardiac rupture (Figure 8, A and B). Furthermore, IL-4 treatment enhanced cardiac function and attenuated post-MI ventricular dilatation (Figure 8, C and D, and Supplemental Tables 2 and 3). Underpinning these benefits, the size of the infarct area was reduced, and the thickness of the infarcted ventricular wall was increased in the IL-4-treated hearts (Figure 9), together with production of more solid and supportive scar tissue. An increased amount and improved organization (alignment) of collagen fibrils in the infarct area were observed (Figure 10A). In contrast, the post-MI increase in collagen deposition in the remote and border areas (representing adverse post-MI ventricular remodeling) was unchanged or attenuated by IL- 4 treatment. Myocardial expression of the Col1a1 and Col3a1 genes on day 7, but not day 28, after MI was upregulated in the IL-4-treated hearts (Figure 10B). Furthermore, an increased number, amplified activation (ratio of $\alpha$ smooth muscle actin ${ }^{+}\left[\alpha \mathrm{SMA}^{+}\right]$Thy $1^{+}$myofibroblasts to Thy $1^{+}$fibroblasts), and attenuated fibroblast apoptosis were noted in the infarct area in the IL-4-treated mice, whereas no alteration in the dynamics of fibroblasts in the remote and border areas was observed (Figure 11 and Supplemental Figure 12). The increased accumulation of M2-like macrophages following IL-4 treatment was also associated with improved capillary formation in the ischemic areas (Supplemental Figure 13), which would increase local perfusion and improve the viability and functionality of fibroblasts and myofibroblasts to enhance the formation of connective tissues.

Improved cardiac repair following IL-4 treatment was primarily achieved via cardiac M2-like macrophages. Several pieces of our data support the idea that the effects of IL-4-induced strength-
Figure 5. Depletion of the post-MI increase in M2-like macrophages in $\mathrm{Trib1}^{-/-}$mice resulted in a critically deteriorated prognosis. (A) The post$\mathrm{Ml}$ survival of mice was dramatically reduced in the $\mathrm{KO}$ group (Trib $1^{1--}$ mice; $n=12$ ) compared with that of the WT group (WT littermates; $n=26$ ), but this effect was fully reversed by transplanting BMDMs in Matrigel (KO plus BMDMs group, $n=10$ ). The control transplantation (KO plus Matrigel group; injection of Matrigel only, $n=10$ ) did not affect the survival rates of the KO group. ${ }^{*} P<0.05$ versus both $\mathrm{KO}$ and $\mathrm{KO}$ plus Matrigel groups, logrank test. (B) The cardiac rupture rate over a 28 -day post-MI period was increased by 9 -fold in the $\mathrm{KO}$ group compared with the rupture rate in the WT group. This fatal event was prevented in the KO plus BMDMs group. The control transplantation (KO plus Matrigel group) did not influence the cardiac rupture rate in the $\mathrm{KO}$ group. $n=$ the same as in $\mathbf{A}$ in each group. $P<0.05$ versus both the KO and KO plus Matrigel groups, $\chi^{2}$ test. (C) Echocardiographic analysis revealed exacerbated cardiac dysfunction in the $\mathrm{KO}$ group, while this deterioration was ameliorated in the $\mathrm{KO}$ plus BMDMs group. $n$ (day 0, day, 7 , and day 28 ) $=12,10$, and 10 in the WT group; 12,5 , and 1 in the KO group; and 10, 6 , and 6 in the KO plus BMDMs group. ${ }^{*} P<0.05$ versus the corresponding time point for the $K O$ group, repeated-measures ANOVA. (Note that the day-28 data were not included in the calculation, because $n=1$ in the $\mathrm{KO}$ group; Trib1/-/- mice rarely survived for 28 days after MI.) LVDd, LV end-diastolic dimension; LVDs, LV end-systolic dimension; LVEF, LV ejection fraction; MG, Matrigel. See Supplemental Table 1 for more detailed echocardiographic data. ening of fibrotic tissue formation described above were more likely to be achieved via cardiac M2-like macrophages rather than through direct effects of IL-4 on fibroblasts or other cell types. First, in Trib1/- mice, in which the post-MI augmentation of M2 macrophages was selectively suppressed, IL-4 treatment did not affect post-MI mortality, cardiac rupture, ventricular function, activation of cardiac fibroblasts, collagen deposition, or capillary density (Figure 12 and Supplemental Table 1). Second, expression levels of IL-4 receptor $\alpha$ (Il4ra) in cardiac fibroblasts were remarkably reduced compared with expression in cardiac M2-like macrophages (Supplemental Figure 14A). Accordingly, our in vitro study showed that IL-4 administration did not activate cultured primary cardiac fibroblasts (Supplemental Figure 14B). Additionally, IL-4 treatment in vivo did not significantly affect collagen deposition, cardiac fibroblasts, or capillary density in the intact hearts of WT mice (Supplemental Figure 15). Taken together, these results suggest that IL-4 treatment increases the number of cardiac M2-like macrophages, which increases the activation of cardiac fibroblasts, enabling them to develop more stable fibrotic tissues to reinforce the fragile, infarcted ventricular wall. This effect improves the post-MI vital and functional prognosis by preventing rupture, dilatation, and dysfunction of the heart.

Cardiac M2-like macrophages activated fibroblasts by secreting $I L-1 \alpha$ and osteopontin. We next used the Boyden chamber system to investigate the mechanisms underlying the ability of M2-like macrophages to regulate fibroblasts in an in vitro setting, in which primary cardiac fibroblasts were cocultured with $\mathrm{CD} 206^{+} \mathrm{F} 4 / 80^{+} \mathrm{CD}_{11 \mathrm{~b}^{+}}$M2-like macrophages isolated from WT mouse hearts (with or without MI) (Figure 13A). Although coculture with M2-like macrophages from intact hearts did not activate fibroblasts, coculture with M2-like macrophages from day-7 post-MI hearts markedly increased fibroblast activation (transformation into $\alpha \mathrm{SMA}^{+}$myofibroblasts; Figure $13, \mathrm{~B}$ and $\mathrm{C}$ ). Given the differences in gene expression in cardiac M2-like macrophages before and after MI (Figure 3, B and C), we hypothesized 
A

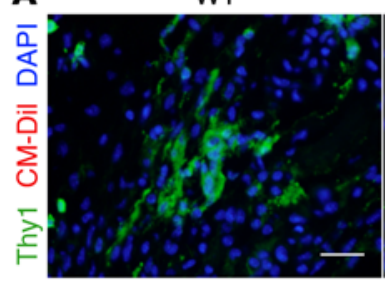

WT

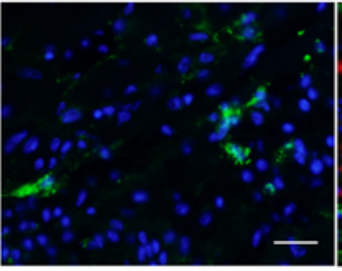

B
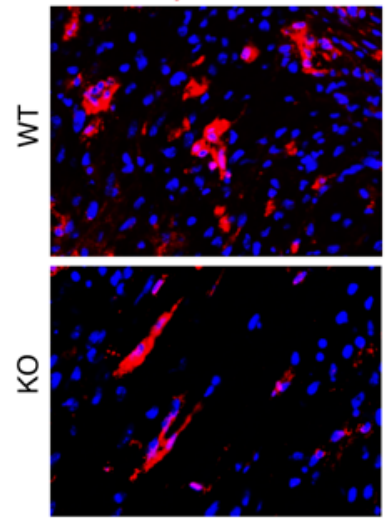

C

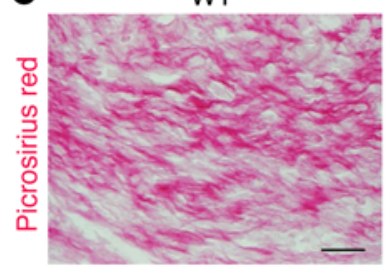

aSMA DAPI

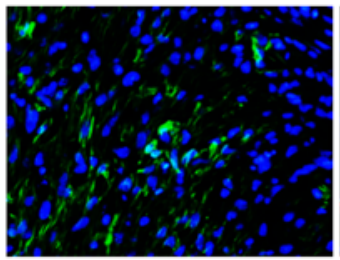

KO

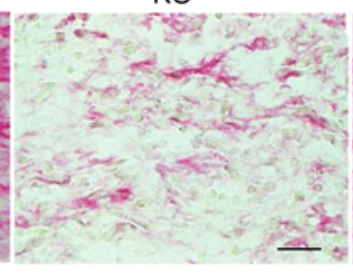

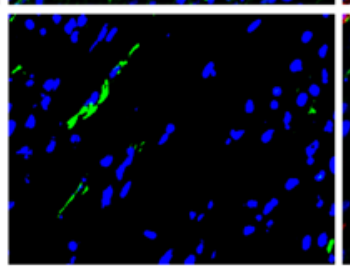

$\mathrm{KO}+\mathrm{BMDMS}$

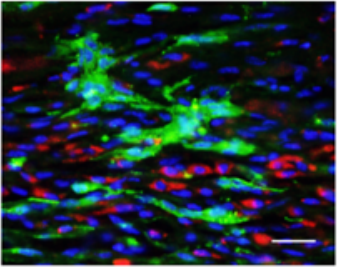

Merge
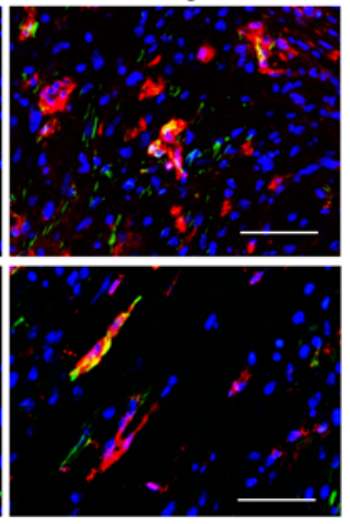

$\mathrm{KO}+\mathrm{BMDMs}$

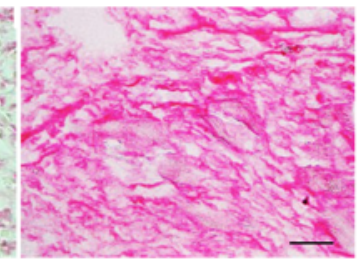

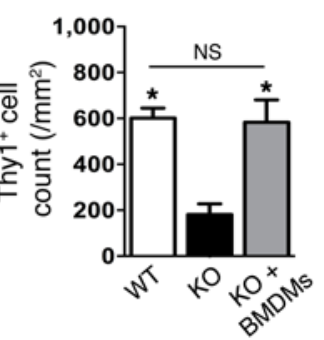

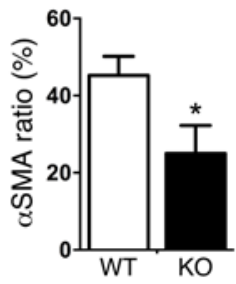

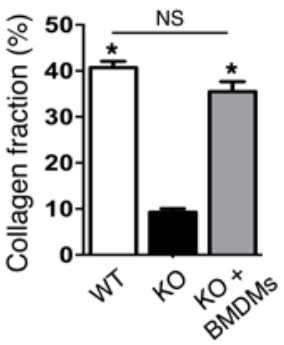

Figure 6. Depletion of M2-like macrophages in Trib1/- mice led to impaired fibroblast-mediated repair of the infarcted myocardium. (A) IHC demonstrated that the accumulation of Thy $1^{+}$fibroblasts in the infarct area at day 7 after MI was largely inhibited in the KO group $(n=5)$ compared with that seen in the WT group $(n=6)$. This deficiency in Trib1 $1^{-1-}$ mice was reversed by BMDM transplantation (KO plus BMDMs group; $n=6$ ). BMDMs were prelabeled with CM-Dil. Scale bars: $50 \mu \mathrm{m}$. ${ }^{*} P<0.05$ versus the KO group, 1 -way ANOVA. (B) Staining for Thy 1 and $\alpha$ SMA in the infarct area on post-MI day 7 indicated that the ratio of $\alpha \mathrm{SMA}^{+}$fibroblasts (percentage of Thy ${ }^{+} \alpha \mathrm{SMA} \mathrm{A}^{+}$myofibroblasts/percentage of Thy ${ }^{+}$fibroblasts) was reduced in the $\mathrm{KO}$ group. Scale bars: $50 \mu \mathrm{m}$. $n=5$ (KO group) and 6 (WT group). ${ }^{*} P<0.05$ versus the WT group, 2-tailed, unpaired Student's $t$ test. (C) Picrosirius red staining showed that the amount (collagen volume fraction) and alignment of collagen fibers in the infarct area on post-MI day 7 was markedly reduced in the KO group ( $n=5$ ) compared with that seen in the WT group $(n=6)$. See Supplemental Figure 10 for additional data in other areas. This impaired tissue repair was reversed in the KO plus BMDMs group $(n=6) .{ }^{*} P<0.05$ versus the KO group, 1-way ANOVA. Scale bars: $50 \mu \mathrm{m}$.

that IL-1 $\alpha(35,36)$ and osteopontin $(37,38)$ might be key mediators of the M2-like macrophage-induced activation of cardiac fibroblasts. Supporting this hypothesis, expression of Illa and Spp1 in the myocardium on day 7 after MI tended to be increased by IL-4 treatment (Supplemental Figure 16A). In contrast, the post-MI upregulation of other profibrotic factors in M2-like macrophages, including Tgfb1 and Pdgfa $(1,2)$, was not significant $(<1.3$-fold compared with that observed in intact hearts). Indeed, Ab-mediated neutralization of IL- $1 \alpha$ and/or osteopontin significantly suppressed the activation of cardiac fibroblast transformation into $\alpha \mathrm{SMA}^{+}$myofibroblasts when the cells were cocultured with postMI M2-like macrophages (Figure 13, B and C, and Supplemental Figure 16B). These data suggest that IL-1 $\alpha$ and osteopontin may act as paracrine mediators that are produced by cardiac M2 macrophages to regulate the activation of cardiac fibroblasts.

\section{Discussion}

Here, we describe the spatiotemporal-specific changes in cardiac M2-like macrophages. The healthy adult murine heart contained

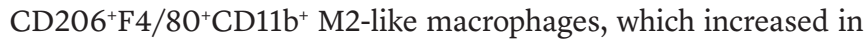
number after MI, peaking on day 7 (the post-MI "healing" stage) predominantly in the infarct area. In parallel, cardiac M2-like macrophages strengthened their tissue-repairing abilities by upregulating various antiinflammatory and repair-associated genes after MI. Consistent with previous reports $(7,8)$, these findings suggested a role for these cells in the repair of infarcted myocardium. To precisely elucidate this role of cardiac M2-like macrophages, we conducted a "specific depletion" study using Trib1/-1 mice. The functional, structural, and histological properties, including the occurrence of M2-like macrophages, of the intact, no-MI hearts of $\mathrm{Trib1}^{-1-}$ mice were all comparable to those of their WT littermates. 

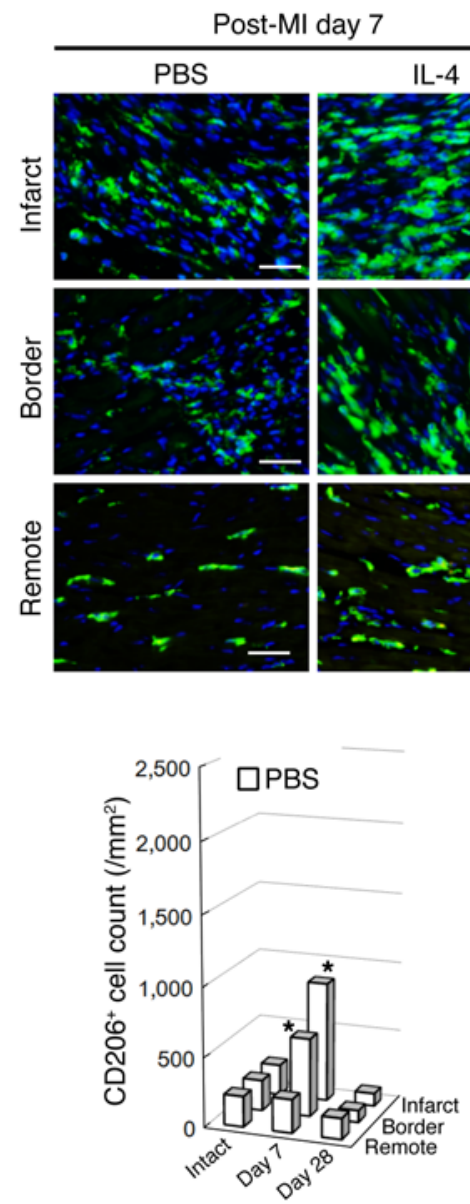

Post-MI day 28

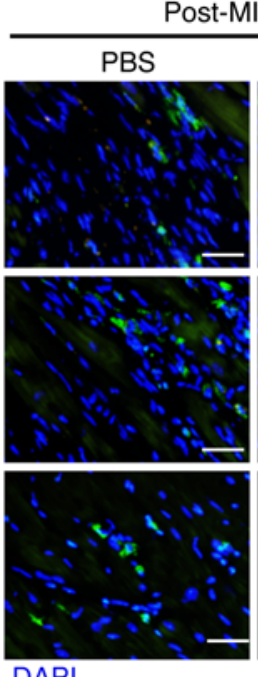

CD206 DAPI

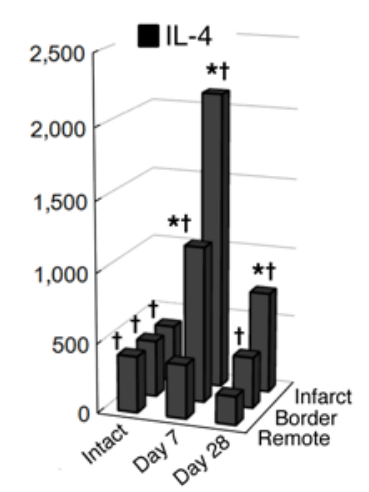

Figure 7. IL-4 administration amplified the post-MI augmentation of cardiac M2-like macrophages in damaged myocardium. Immunofluorescence revealed that the post-MI augmentation of $\mathrm{CD}_{206} \mathrm{C}^{+} \mathrm{M2}$-like macrophages in the border and infarct areas of the PBS-injected group ( $n=6$ in each point) was further amplified in the IL-4treated group ( $n=6$ in each point). Scale bars: $50 \mu \mathrm{m}$. ${ }^{*} P<0.05$ versus intact, no-MI hearts in each group; ${ }^{\dagger} P<0.05$ versus PBS-treated group at the corresponding time and area; repeated-measures ANOVA.
However, these mice exhibited a complete loss of the post-MI augmentation of M2-like macrophages in the heart, without affecting other cell types including proinflammatory monocytes and macrophages. This model allowed us to dissect the precise role of M2-like macrophages after MI without bias from other subsets of macrophages, which is different from previous models using pan-macrophage depletion via techniques such as clodronate liposome injection (7-9). The results were dramatic: the selective suppression of M2-like macrophages after MI resulted in a largely reduced survival rate, with 9-fold more frequent cardiac ruptures and exacerbated cardiac dysfunction than occurred in their WT littermates. This result corresponded to a defective formation of fibrotic connective tissues in the infarct area, along with impaired accumulation and activation of cardiac fibroblasts. This finding was strengthened by an additional rescue experiment, in which M2-like macrophages were supplied externally by transplanting BMDMs collected from WT mice. The transplanted macrophages exhibited an M2 phenotype in the damaged myocardium, achieving a post-MI frequency of M2-like macrophages equivalent to that in WT mice, which is consistent with findings in the lung (39). Notably, this recovery of M2-like macrophages successfully rescued the poor post-MI prognosis and reduced tissue repair in Trib1/-/ mice. This series of studies using $\mathrm{Trib1}^{-/-}$mice provides evidence for the essential role of cardiac M2-like macrophages in fibroblast-mediated repair of the infarcted, fragile myocardium via the formation of secure fibrotic tissues. This repair allows a certain degree of maintenance (imper- fect, but almost sufficient to survive) of the geometry, integrity, and function of the heart after MI.

In addition, we demonstrated that external IL-4 stimulation amplified the post-MI augmentation of cardiac M2-like macrophages in WT mice. IL-4 treatment substantially strengthened the formation of connective tissues in the infarcted myocardium, which was underpinned by increased accumulation, amplified transformation into myofibroblasts, and attenuated apoptosis of fibroblasts. This "physiological" fibrotic tissue formation resulted in an improved post-MI prognosis and cardiac structure and function. In contrast, the extracellular collagen deposition in the remote and border areas, which represents adverse ventricular remodeling, was not increased by IL-4 treatment, alleviating concerns that excessive, pathological myocardial fibrosis would be induced by this treatment. Our results also demonstrated an insignificant response of cardiac fibroblasts to IL-4 stimulation in vitro, a markedly reduced expression of Il4ra in cardiac fibroblasts compared with expression in cardiac M2-like macrophages, and an absence of IL-4-induced repair in $\mathrm{Trib1}^{-/-}$mice, in which M2 macrophages, but not fibroblasts, were selectively depleted. All of these data suggested that the observed IL-4-induced activation of cardiac fibroblasts was mainly mediated by M2-like macrophages, rather than a direct effect of IL-4 stimulation on fibroblasts. Investigations using cell type-specific conditional Il4ra-KO mice will be required to further confirm the direct role of IL-4 in cardiac M2-like macrophages. Collectively, our results from the study of 

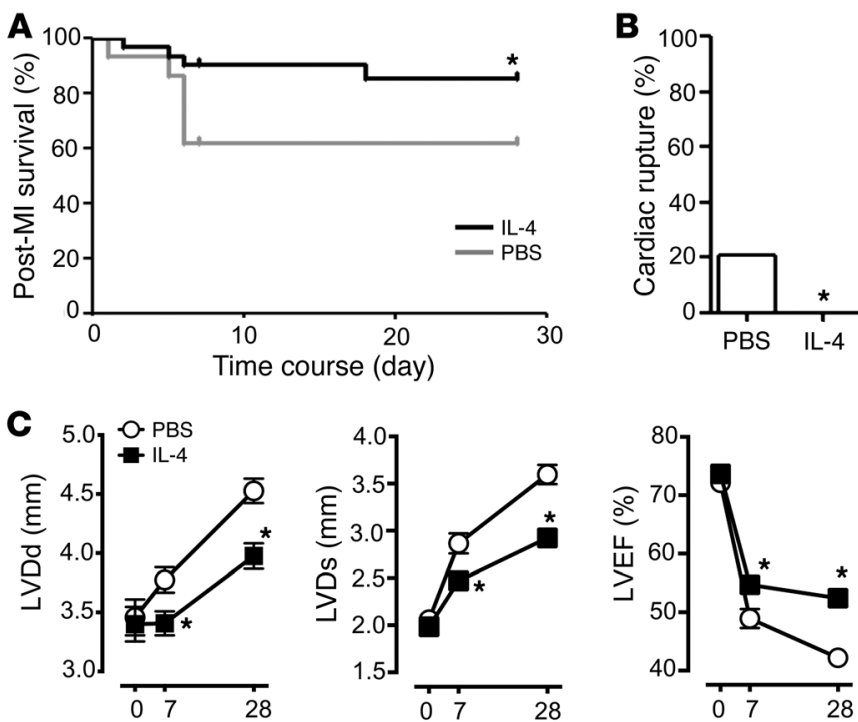

Time course (day)
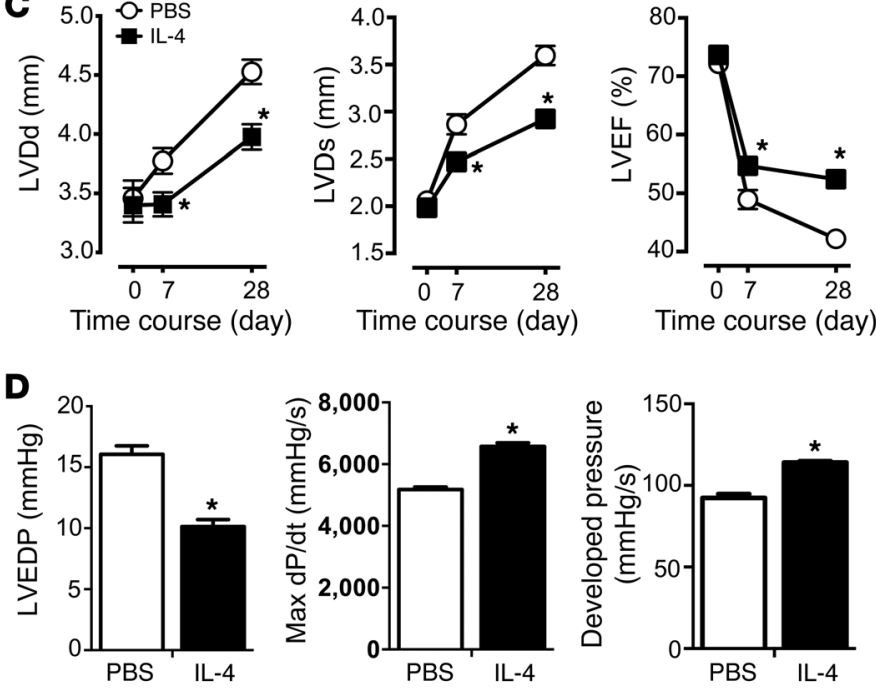

selective depletion followed by rescue and the specific augmentation experiment indicated that cardiac M2 macrophages govern the fibroblast-mediated repair of infarcted myocardial tissue by forming secure fibrotic tissues, which critically determines the overall post-MI prognosis.

As regards the mechanism underlying M2-like macrophagemediated cardiac repair, the coculture of primary cardiac fibroblasts and isolated cardiac M2-like macrophages indicated that M2-like macrophages from infarcted heart, but not those from nor-
Figure 8. IL-4 treatment improved post-MI prognosis and cardiac function. (A) Post-MI survival rates of the mice were improved in the IL-4-treated group $(n=31)$ compared with those of the PBS-treated group $(n=29) .{ }^{*} P<0.05$ versus the PBS-treated group, log-rank test. (B) The cardiac rupture rate over a 28-day post-MI period was reduced in the IL-4-treated group ( $n=31)$ compared with that of the PBS-treated group $(n=29) .{ }^{*} P<0.05$ versus the PBS-treated group, $\chi^{2}$ test. (C) Echocardiographic analysis revealed that the IL-4-treated group showed improved cardiac function and attenuated ventricular dilatation compared with the PBS-treated group. $n=12$ for each point in each group. ${ }^{*} P<0.05$ versus the PBS-treated group at the corresponding time point, repeatedmeasures ANOVA. See Supplemental Table 2 for additional pre- and post-MI echocardiographic data. (D) Cardiac catheterization assessments detected improved hemodynamics and enhanced cardiac performance at day 28 after MI in the IL-4-treated group $(n=10)$ compared with the PBS-treated group $(n=10) .{ }^{*} P<0.05$ versus the PBS-treated group, 2-tailed, unpaired Student's $t$ test. See Supplemental Table 3 for more detailed data. dP/dt, LV contractility; LVEDP, LV end-diastolic pressure; Max, maximum. mal heart, were able to increase the activation of cardiac fibroblast transformation into myofibroblasts. Illa and $s p p 1$ (osteopontin) expression was upregulated by more than 3-fold in M2-like macrophages from the MI heart compared with those from the normal heart. In contrast, other known fibrotic factors, including Tgfb1 and Pdgfa, were not significantly upregulated. Indeed, the studies with neutralizing Abs confirmed that IL-1 $\alpha$ and osteopontin are essential mediators of M2-like macrophage-induced activation of fibroblasts, at least in vitro. These data provide new biological insights
A

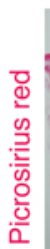

PBS

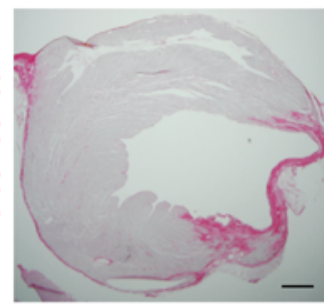

Figure 9. IL-4 treatment reduced infarct size and increased infarcted wall thickness. Picrosirius red staining of day-28 post-MI heart samples indicated that the infarct size was reduced $(\mathbf{A})$ and the thickness of the walls of the infarct area was increased (B) in the IL-4-treated group compared with the PBS-treated group. Scale bars: $1 \mathrm{~mm} . n=6$ in each group. ${ }^{*} P<0.05$ versus the PBS-treated group, 2-tailed, unpaired Student's $t$ test.
B

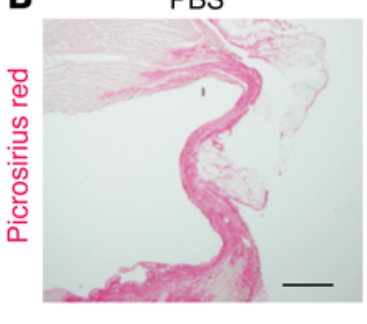

$\mathrm{IL}-4$
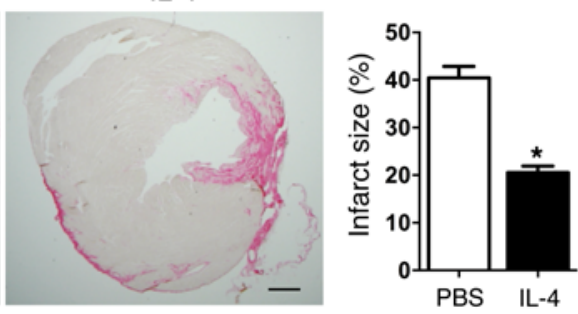

$\mathrm{IL}-4$

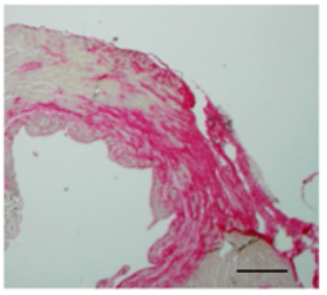


A

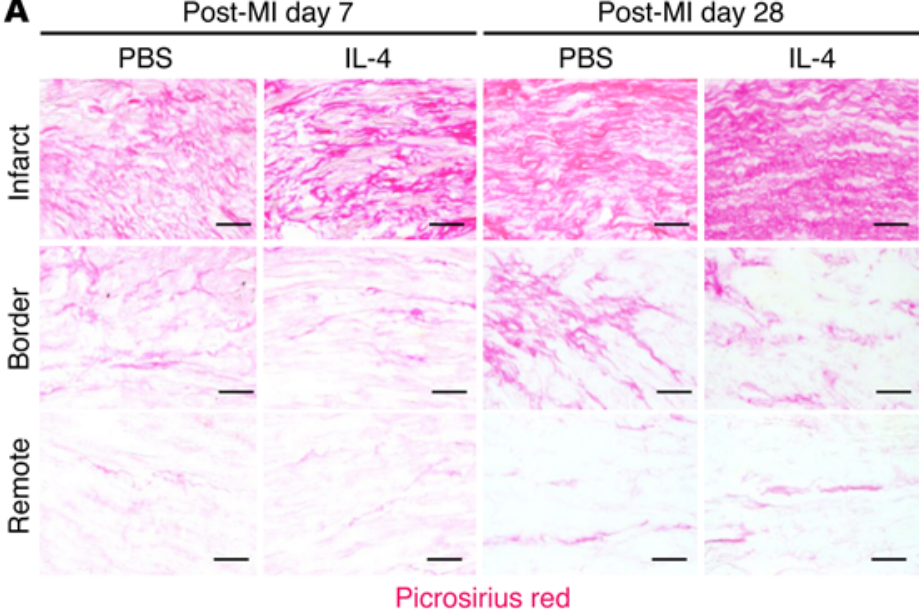

Figure 10. IL-4 treatment enhanced the formation of connective tissue in the infarct area. (A) Picrosirius red staining revealed that formation of fibrotic tissues in the infarct area was increased in the IL-4-treated group compared with that seen in the PBS-treated group. Results in the graphs are presented as the collagen volume fraction (percentage). In contrast, extracellular collagen deposition in the remote and border areas was unchanged or attenuated in the IL-4-treated group. For images of the intact, no-MI hearts, see Supplemental Figure 15. Scale bars: $100 \mu \mathrm{m} . n=6$ for each point in each group. ${ }^{*} P<0.05$ versus the intact, no-Ml hearts in each group; ${ }^{\dagger} P<0.05$ versus the WT group at the corresponding time and area; repeated-measures ANOVA. (B) qRT-PCR indicated that the expression of collagen genes was upregulated in the hearts of the IL-4-treated group at post-MI day 7 but not day 28 compared with the PBS-treated group. $n=6$ for each point in each group. ${ }^{*} P<0.05$ versus the corresponding time point of the PBS-treated group, repeated-measures ANOVA.

The "original" cardiac-resident M2-like macrophages in intact adult hearts primarily originate from the yolk sac, and upon MI injury, most of these cells disappear (via cell death and/or migration out) by day 1 after MI (7). We confirmed this attrition of original cardiac M2-like macrophages early after MI (Supplemental Figure 17). Subsequently, "new" M2-like macrophages develop in the MI heart, predominantly originating from the BM (7-9). Therefore, the major subsets of pre- and post-MI cardiac M2-like macrophages are likely to have different origins and thus may utilize different mechanisms (different roles of and/or different compensatory pathways to TRIB1) for their differentiation.

Although the cellular mechanism by which Trib1 deficiency results in a reduced frequency of M2-like macrophages after MI remains uncertain, the previously reported reduced differentiation of BM cells into M2-like macrophages (16) may be involved. In addition, the reduced postMI proliferation of cardiac M2-like macrophages in Trib1 ${ }^{-1-}$ mice (Supplemental Figure 5) may also contribute to the reduced post-MI augmentation of these macrophages. The influence of Trib1 deficiency on the migration and recruitment of BM-derived M2-like macrophages and/or their precursors remains to be determined. Given that the recruitment of M1-like macrophages and monocytes from the circulation was not reduced in Trib1-- mice (ref. 16 and Supplemental Figure 7) and that some of cardiac M2-like macrophages share the same origin with M1-like macrophages and monocytes (Ly6 $\mathrm{C}^{+}$monocytes), we hypothesize that the migration of the precursors of BM-derived M2-like macrophages might not be severely affected. However, it is not currently feasible to prove this hypothesis, because the precise identification of the circulating precursors of cardiac M2-like macrophages remains uncertain.

On the other hand, IL-4 treatment increased CD206 ${ }^{+}$cardiac M2-like macrophages both before and after MI. We believe that the pre-MI increase depends on amplified local proliferation of the original cardiac-resident M2-like macrophages. Previous studies have demonstrated that IL-4 treatment is sufficient to increase the number of M2-like macrophages through local proliferation (23, 24). Consistent with this observation, our study (Ki67 staining) revealed an increase in the local proliferation of $\mathrm{CD}^{2} \mathrm{O6}^{+}$cardiac 
A
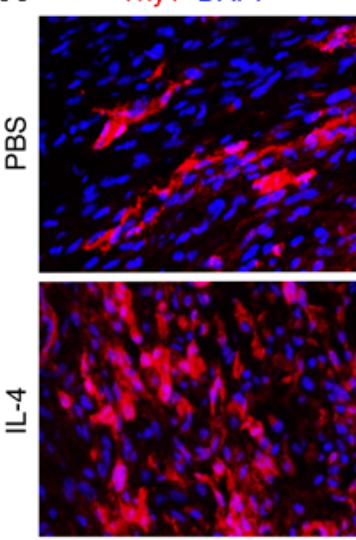

B
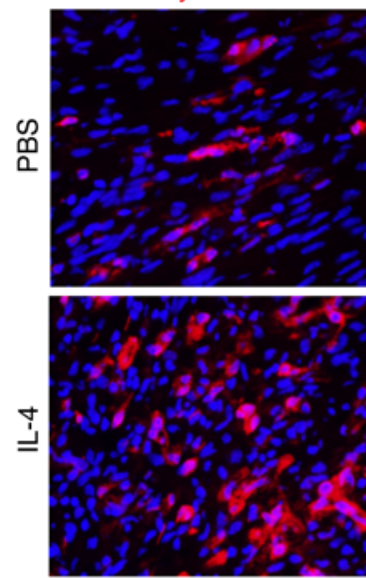

aSMA DAPI
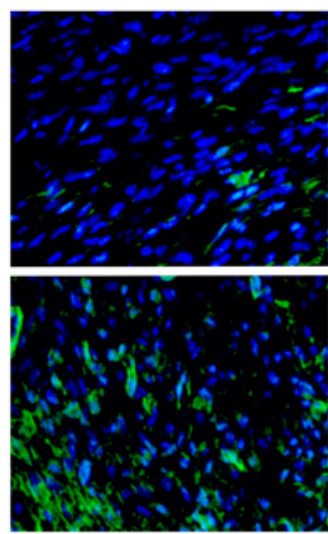

c-caspase 3 DAPI
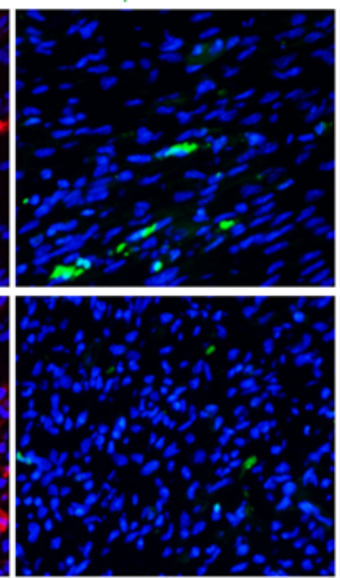

Merge
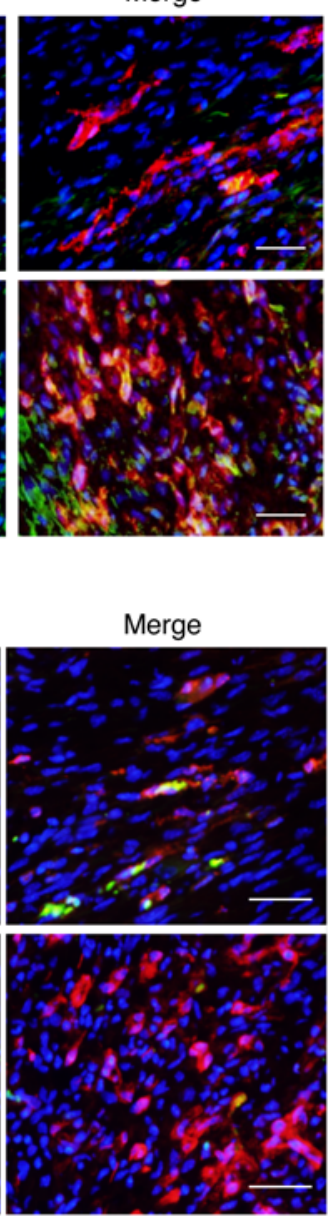
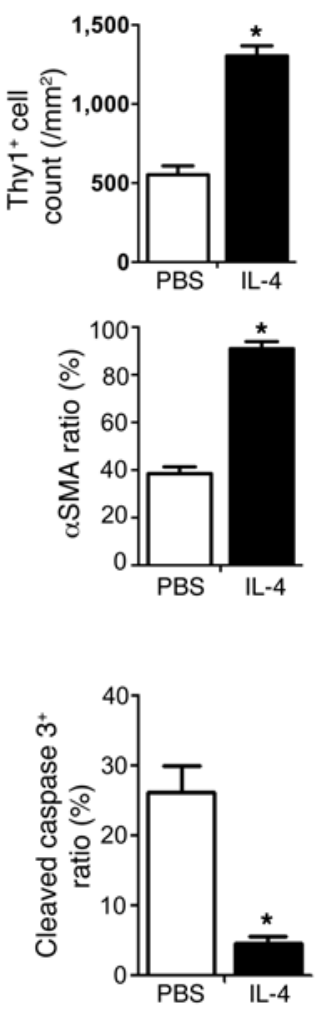

Figure 11. IL-4 treatment increased the post-MI number and activation of cardiac fibroblasts, with attenuated apoptosis. (A) IHC demonstrated that the number and activation (transformation into Thy $1^{+} \alpha S M A^{+}$myofibroblasts) of Thy $1^{+}$cardiac fibroblasts in the infarct area of the IL-4-treated group were increased at post-MI day 7 compared with the PBS-treated group. Scale bars: $50 \mu m . \alpha S M A$ ratio is the percentage of $\alpha S M A^{+}$Thy $1^{+}$myofibroblasts to the percentage of Thy ${ }^{+}$fibroblasts. $n=6$ different hearts in each group. ${ }^{*} P<0.05$ versus the PBS-treated group, 2 -tailed, unpaired Student's $t$ test. See Supplemental Figure 7 for additional data in other areas and at other time points. (B) Double immunofluorescence staining for Thy 1 and cleaved caspase 3 demonstrated that apoptosis of cardiac fibroblasts in the infarct area of the IL-4-treated group was attenuated at post-MI day 7 compared with the PBStreated group. Scale bars: $50 \mu \mathrm{m} . n=6$ in each group. ${ }^{*} P<0.05$ versus the PBS-treated group, 2 -tailed, unpaired Student's $t$ test.

M2-like macrophages in non-MI hearts following IL-4 treatment. In contrast, the augmented post-MI increase in the number of $\mathrm{CD}_{20}{ }^{+}$cardiac M2-like macrophages following IL-4 treatment is likely to be underpinned by the increased differentiation and local proliferation of BM-derived M2-like macrophages. It has been repeatedly reported that IL-4 stimulates the differentiation of BMDMs into an M2-like phenotype $(25,26)$. Our study (Ki67 staining) revealed that the increased proliferation of CD206 $6^{+}$cardiac M2-like macrophages in post-MI hearts was further amplified by IL-4 treatment. Given our data showing that the original cardiac M2-like macrophages mostly disappeared early after MI, even following IL-4 treatment (Supplemental Figure 17B), it is unlikely that the original yolk-sac-derived cardiac-resident M2-like macrophages significantly contributed to the post-MI increase in $\mathrm{CD}^{2} \mathrm{O6}^{+}$cardiac M2-like macrophage numbers following IL-4 treatment. Further investigations are needed to fully elucidate these speculated mechanisms and determine the detailed dynamics of cardiac macrophages following Trib1 KO or IL-4 treatment.
We used the intrapericardial injection method to deliver BMDMs (incorporated in Matrigel) into the heart after MI. This might not be a standard method, but it reproducibly resulted in the successful accumulation of BMDMs in the infarcted myocardium. One concern associated with this method is that the procedure itself (transplantation of Matrigel without BMDMs) might affect the post-MI survival or development of cardiac rupture in Trib1/mice, but we confirmed that this was not the case (Figure 5, A and B). Although an i.v. injection of BMDMs may be more technically straightforward, our previous study revealed that this cell-delivery method did not result in a sufficient post-MI engraftment of BMDMs into the heart (data not shown).

It is reasonable to suggest that strategies that enhance M2 macrophage-mediated cardiac repair will be useful for the treatment of MI, which remains a major cause of human death and disability (17). The therapeutic effect of this approach is based on enhancement of the intrinsic self-repair system that is mediated by naturally occurring M2 macrophages, which activate multiple 
A

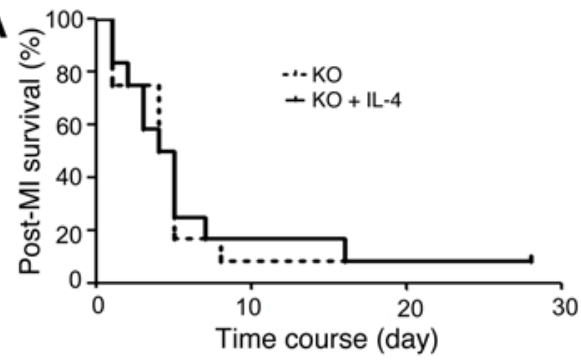

B

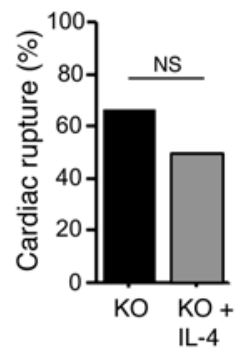

C
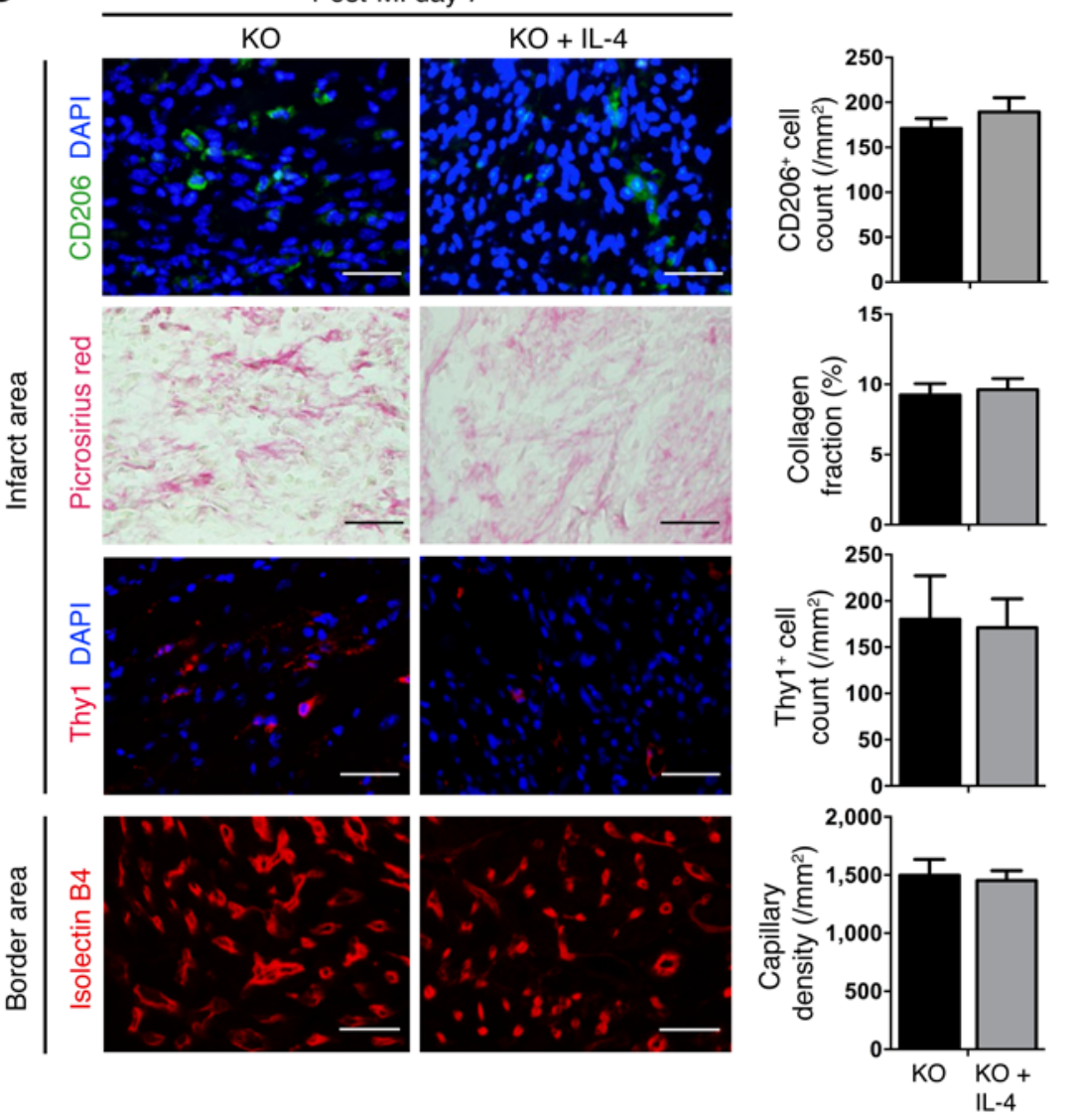

Figure 12. IL-4-induced myocardial repair was abolished in Trib1/- mice. (A) The post-MI survival rate of Trib1 $^{-1-}$ mice (KO group) was not improved following IL-4 treatment (KO plus IL-4-treated group). $n=12$ in each group; log-rank test. (B) The cardiac rupture rate over a 28-day post-MI period was not significantly changed between the $\mathrm{KO}$ and $\mathrm{KO}$ plus IL-4-treated groups. $n=12$ in each group; $\chi^{2}$ test. (C) Histological studies showed that the frequency of $\mathrm{CD} 206^{+} \mathrm{M} 2$-like macrophages, collagen fiber formation, and accumulation of Thy ${ }^{+}$ fibroblasts in the infarct area and the density of isolectin $\mathrm{B}^{+}$capillary vessels in the border area at post-MI day 7 were equivalent between the $\mathrm{KO}$ and $\mathrm{KO}$ plus IL-4-treated groups. Scale bars: $50 \mu \mathrm{m} . n=3$ in each group; 2-tailed, unpaired Student's $t$ test.

fully proven) $(43,44)$. In addition, our study did not observe any evident adverse effect of IL-4, including delayed healing or excessive fibrotic scar formation of anastomosed skin. Nevertheless, additional preclinical studies are required to completely address the safety issues surrounding IL-4 treatment. Local targeted delivery of IL-4 to the heart through an intracoronary injection or use of biomaterials for controlled drug delivery may ease the safety concerns.

In conclusion, our data provide evidence that cardiac M2-like macrophages critically govern the activity of fibroblasts and myofibroblasts in the infarcted adult murine heart. This process plays a vital role in the repair of the infarcted, fragile ventricular wall by forming firm fibrotic tissues. This tissue repair mechanism controls the degree of maintenance of the rigidity and function of the infarcted heart, thus determining the post-MI prognosis by preventing cardiac rupture and attenuating the development of heart failure. It was suggested that IL-1 $\alpha$ and osteopontin might play a role in the regulation of fibro-

repair mechanisms, and is therefore natural, dynamic, and effective. Although this study focused on fibroblast-mediated tissue repair, it is likely that M2-like macrophages mediate supplementary benefits for post-MI cardiac repair, including reduced inflammation, protection of cardiomyocytes, and neovascular formation, as our data revealed an upregulation of the relevant repair-associated genes in these cells after MI. Our results suggest that IL-4 administration has the potential to accomplish such a therapeutic strategy. Of course, additional translational and preclinical studies must be successfully completed before this treatment is applied to patients. In particular, a safety concern might be posed by the possibility that elevated levels of circulating IL-4 might cause side effects, e.g., exacerbation of allergic diseases, atherosclerosis, or fibrosis $(40-42)$. However, these possibilities may be overstated in rodent or cell models, because the clinical safety of IL-4 injections (either i.v. or s.c.) has been extensively reported in previous clinical trials (more than 500 patients have been tested), all of which were aimed at cancer therapy (the anticancer effect was not blasts by cardiac M2-like macrophages. In addition, the targeted activation of M2-like macrophages following IL-4 administration enhances this endogenous repair mechanism, suggesting that this approach may have therapeutic potential for the treatment of MI.

\section{Methods}

Animals. Six- to eight-week-old mice were used in the experiments. C57BL/6 mice were purchased from Charles River Laboratories. Trib1 $1^{-1-}$ mice were a gift of Shizuo Akira (Osaka University, Osaka, Japan) and were a hybrid of a C57BL/6 and SV129 mixed background (16). Age-matched WT littermates were used as a control. The mice were maintained in a specific pathogen-free room in our animal facility on a 12-hour light/12-dark cycle with free access to food and water. The mice were randomly assigned to different groups, and, where possible, the in vitro studies and in vivo procedures and assessments were performed in a blinded manner.

Induction of MI. MI was induced in mice by ligating the left coronary artery under $1.0 \%$ isoflurane anesthesia and mechanical venti- 
A
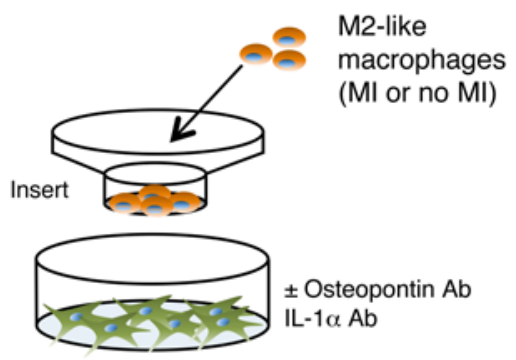

Cardiac fibroblasts
B

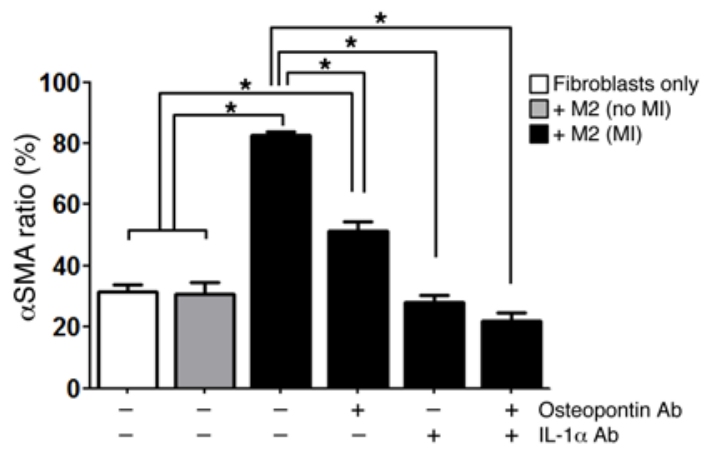

C Fibroblasts
only
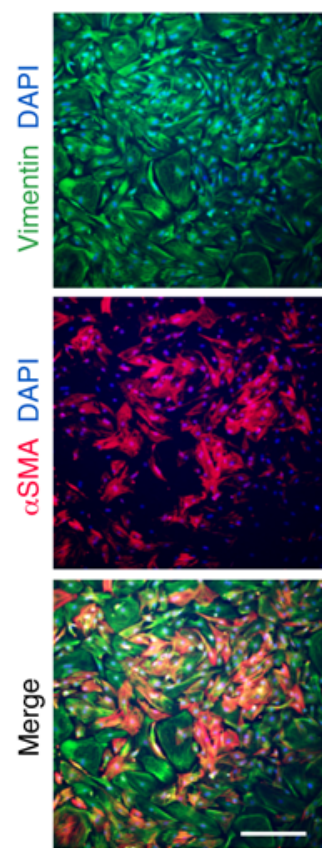

Fibroblasts + M2 (no Ml)
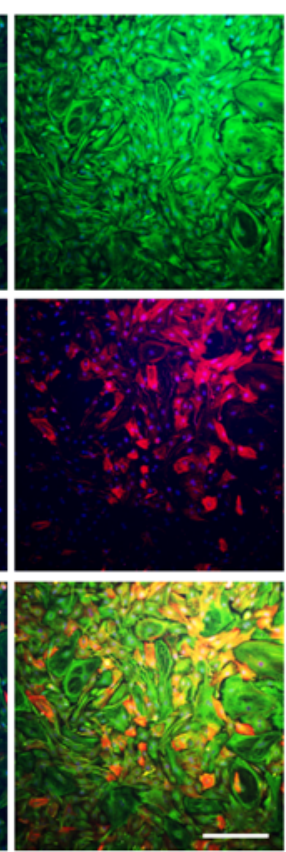

Fibroblasts + M2 (MI)

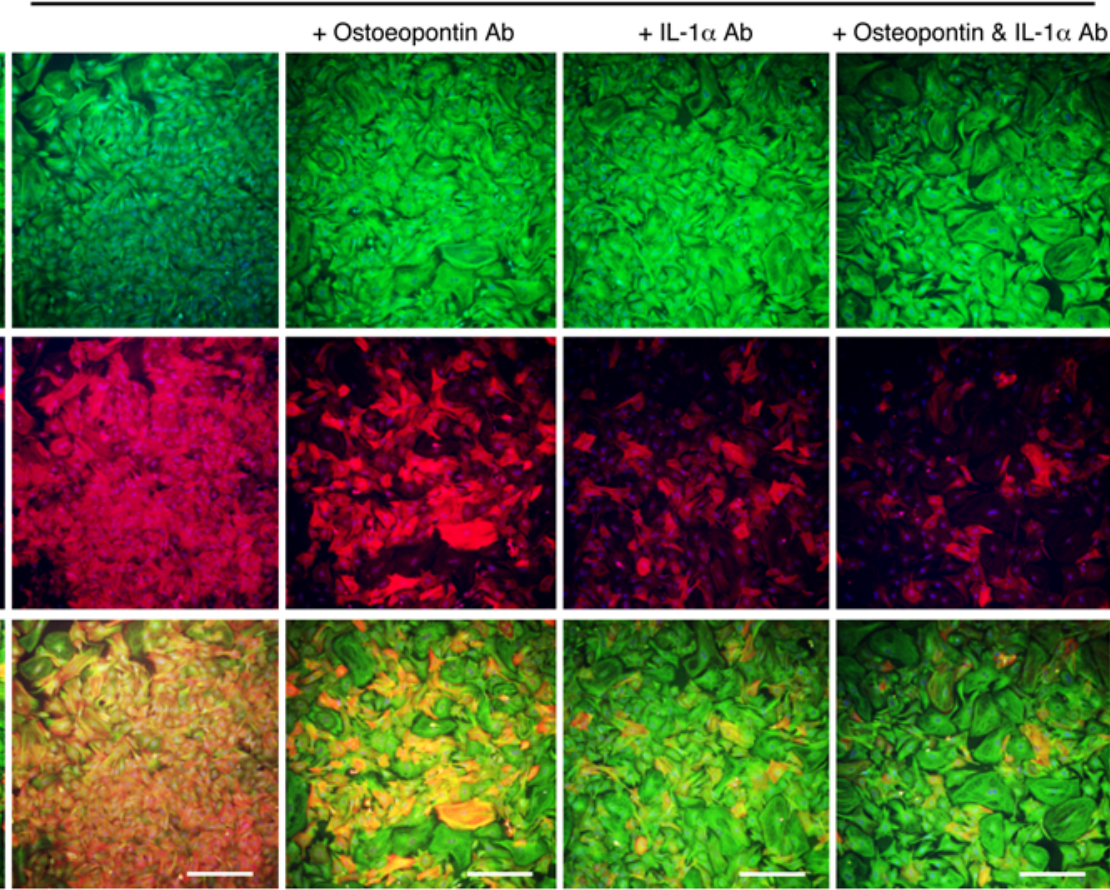

Figure 13. IL-1 $\alpha$ and osteopontin played a vital role in cardiac M2-like macrophage-induced fibroblast activation. (A) Schematic of the coculture experimental protocol. Primary cardiac fibroblasts were cocultured with or without CD206+F4/80+CD11 b+ M2-like macrophages from intact, no-MI or MI (day 7 after MI) hearts in the Boyden chamber culture system for 48 hours. Neutralizing Abs against osteopontin and/or IL-1 $1 \alpha$ were added to the relevant groups at the beginning of the coculture with $\mathrm{M2}$-like macrophages. (B) Activation of cardiac fibroblasts (ratio of vimentin $\alpha \mathrm{SMA}^{+}$myofibroblasts to vimentin ${ }^{+}$ fibroblasts) was increased in the cocultures with $\mathrm{M} 2$ (MI) macrophages but not with $\mathrm{M} 2$ (no $\mathrm{MI}$ ) macrophages. Representative images are presented in C. This increased activation was eliminated by neutralizing Abs against osteopontin and IL-1 $\alpha . n=8-10$ in each group. ${ }^{*} P<0.05,1$-way ANOVA. (C) Representative images of immunocytochemical staining for vimentin and $\alpha$ SMA. Nuclei were counterstained with DAPI. Scale bars: $200 \mu \mathrm{m}$.

lation, as previously described (45). The successful generation of MI was confirmed by changes in the color and motion of the LV walls. The survival rate after MI was monitored daily.

IL-4 administration. IL-4c (5 $\mu$ g recombinant IL-4 [PeproTech; 214-14] and $25 \mu \mathrm{g}$ neutralizing monoclonal anti-IL-4 Ab [BD Biosciences; 554387]) (23) dissolved in $100 \mu \mathrm{l}$ PBS was injected twice (2 days before and immediately before the induction of MI) into the peritoneal cavity of the mice. An equivalent volume of PBS was similarly injected as a control.

Isolation of heart cells. Mouse heart cells were isolated as previously described (46), with some modifications. Immediately after cervical dislocation, the aorta was clamped, and cooled HBSS (Sigma-Aldrich) was injected into the LV cavity. The isolated hearts were cut into 1 -mm pieces, digested with $0.05 \%$ collagenase II (Sigma-
Aldrich) at $37^{\circ} \mathrm{C}$ for 15 minutes, and filtered using a $40-\mu \mathrm{m}$ cell strainer (BD Falcon). The remnant heart tissues were again digested with a fresh digestion solution and filtered similarly; this cycle was repeated 5 times. The suspension obtained at each cycle was combined and subjected to flow cytometric analyses or FACS after erythrocytes were depleted using Red Cell Lysis Buffer (BioLegend) according to the manufacturer's protocol.

Flow cytometry and FACS. The isolated cells were resuspended in FACS buffer (HBSS plus $2 \mathrm{mM}$ EDTA plus 0.5\% BSA) and preincubated with an anti-mouse CD16/CD32 Ab (rat, 1:100 dilution; eBioscience; catalog 14-0161) to block the Fc receptor. The dead cells and debris were excluded by forward scatter/side scatter (FSC/SSC) and DAPI staining (1:1,000 dilution; Sigma-Aldrich). To determine their phenotypes, the cells were stained with the following Abs for 3 hours at 
$4^{\circ} \mathrm{C}$ : APC-conjugated anti-CD11b Ab (rat, 1:100 dilution; eBioscience; catalog 17-0112); phycoerythrin- (PE-) or Alexa Fluor 488-conjugated anti-F4/80 Ab (rat, 1:20 dilution; eBioscience; catalog 12-4801 or 53-4801); Alexa Fluor 488-conjugated anti-CD206 Ab (rat, 1:50 dilution; BioLegend; catalog 141709); PerCP-conjugated anti-CD11c $\mathrm{Ab}$ (hamster, 1:20 dilution; BioLegend; catalog 117326); allophycocyanin- (APC-) or PE-conjugated anti-CCR2 Ab (rat, 1:20 dilution; R\&D Systems; catalog FAB5538A or FAB5538P); and APC- or PerCP-conjugated anti-Ly6C Ab (rat, 1:25 dilution; eBioscience; catalog 17-5932 or 45-5932). Flow cytometric analyses were performed using FACSCalibur flow cytometer (BD Biosciences) and FlowJo software (Tree Star). Cell sorting was performed using a FACSAria II (BD Biosciences).

Isolation of peritoneal macrophages. Mouse peritoneal cells were collected as previously described (47). Immediately after cervical dislocation, $5 \mathrm{ml}$ cold HBSS was injected into the peritoneal cavity and was retrieved into RPMI 1640 medium with 10\% FBS. Erythrocytes were lysed with Red Cell Lysis Buffer (BioLegend) according to the manufacturer's protocol, and the resulting cell suspension was subjected to FACS, as described above.

Preparation of BMDMs. Mouse BMDMs were prepared from the femurs and tibiae of 8-week-old WT mice as previously described (39). BM mononuclear cells were collected by centrifugation on FicollPaque (GE Healthcare) and were cultivated in DMEM (Sigma-Aldrich) containing $10 \% \mathrm{FBS}, 50 \mathrm{U} / \mathrm{ml}$ penicillin, $50 \mu \mathrm{g} / \mathrm{ml}$ streptomycin, and $10 \mathrm{ng} / \mathrm{ml}$ granulocyte-macrophage CSF (GM-CSF) (R\&D Systems; 415-ML) overnight in a $\mathrm{CO}_{2}$ incubator. The non- or weakly attached cells were collected and transferred to new dishes and cultivated for an additional 5 days. The cells were prepared for immunocytochemistry or transplantation. Immunocytochemical staining was performed to evaluate monocyte-to-macrophage differentiation. The attached cells were fixed with $4 \%$ paraformaldehyde (PFA) (Sigma-Aldrich) in PBS for 5 minutes. The cells were blocked in $5 \%$ goat serum in PBS for 30 minutes. The samples were incubated with the following primary Abs overnight at $4^{\circ} \mathrm{C}$ : anti-CD68 Ab (1:100 dilution; AbD Serotec; catalog MCA1957); PE-conjugated anti-CD14 Ab (1:20 dilution; eBioscience; catalog 12-0141); and PE-conjugated anti-F4/80 Ab (1:20 dilution; eBioscience; catalog 12-4801). CD68 was visualized by incubating the cells with an Alexa Fluor 488-conjugated secondary Ab (1:300 dilution; Invitrogen; catalog A-11006) for 1 hour at room temperature. DAPI (1:500 dilution; Sigma-Aldrich) was used to stain the nuclei for 1 hour at room temperature. The images were acquired with an All-inOne microscope (Keyence). Immunocytochemical results are shown in Supplemental Figure 18A. Most of the produced cells were positive for CD68, CD14, and F4/80.

$B M D M$ transplantation. For histological tracing after transplantation, BMDMs were labeled with CM-DiI (Molecular Probes) as previously described (48). CM-DiI-labeled BMDMs $\left(2 \times 10^{5}\right.$ cells $)$ in 25 $\mu \mathrm{l}$ PBS were suspended in $25 \mu \mathrm{l}$ Matrigel (Corning). Shortly after left coronary artery ligation (as described above, but without opening the pericardium), the BMDM-containing Matrigel was injected into the pericardial cavity. A scheme describing the BMDM transplantation is shown in Supplemental Figure 18B.

Isolation of cardiac fibroblasts. Cardiac fibroblasts were isolated from C57BL/6 mice (Charles River Laboratories) as previously described (49). The isolated mouse heart was cut into 1-mm pieces, and the fragments were plated evenly in $10-\mathrm{cm} 0.1 \%$ gelatin-coated dishes so that they did not contact each other. Each fragment was cov- ered by a droplet of RPMI1640 medium containing 10\% FBS, 50 U/ $\mathrm{ml}$ penicillin, and $50 \mu \mathrm{g} / \mathrm{ml}$ streptomycin and incubated for 24 hours at $37^{\circ} \mathrm{C}$ in a $\mathrm{CO}_{2}$ incubator. Then, a sufficient amount of medium was added to cover the entire bottom of the culture dish, and the culture was incubated for an additional 24 hours. Sufficient medium was then added to completely cover the heart fragments, followed by an additional 5 days of culture. The cells were collected by trypsinization when fibroblast outgrowth was observed. The fibroblasts were maintained in RPMI 1640 medium containing 10\% FBS, $50 \mathrm{U} / \mathrm{ml}$ penicillin, and $50 \mu \mathrm{g} / \mathrm{ml}$ streptomycin in $0.1 \%$ gelatin-coated culture flasks. The cells were used at passage 3 or 4 .

Coculture of cardiac fibroblasts and M2-like macrophages in a Boyden chamber. Cardiac fibroblasts $\left(2 \times 10^{4}\right)$ were plated on a $0.1 \%$ gelatin-coated 6-well dish (Thermo Scientific) and cultured in RPMI 1640 medium containing 10\% FBS, $50 \mathrm{U} / \mathrm{ml}$ penicillin, and $50 \mu \mathrm{g} /$ $\mathrm{ml}$ streptomycin. After 48 hours, $2 \times 10^{4} \mathrm{M} 2$-like macrophages were seeded onto polycarbonate membrane inserts (pore size, $0.4 \mu \mathrm{m}$; Thermo Scientific), which were set into the wells containing fibroblasts. Neutralizing anti-osteopontin Ab $(10 \mu \mathrm{g} / \mathrm{ml}$; R\&D Systems; catalog AF808) and/or neutralizing anti-IL-1 $\alpha$ Ab $(10 \mu \mathrm{g} / \mathrm{ml}$; R\&D Systems; catalog MAB400) was added to the culture medium at the beginning of coculture. After 48 hours of coculture, fibroblasts were prepared for the analysis.

Routine analysis methods. Detailed protocols of routine research methods including cardiac function measurement, RNA extraction and real-time PCR, microarray analysis, IHC, Picrosirius red staining, and immunocytochemistry are described in the Supplemental Methods.

Statistics. All statistical tests were performed using GraphPad Prism software, version 6 (GraphPad Software). Data represent the mean \pm SEM. For comparisons between multiple groups, repeatedmeasures ANOVA or 1- or 2-way ANOVA was performed, followed by Bonferroni's post-hoc test. Two groups were compared using the 2-tailed, unpaired Student's $t$ test. Cardiac rupture rates were compared with the $\chi^{2}$ test, whereas the survival curves were compared with the log-rank test.

Study approval. The investigations using animals conformed to the Principles of Laboratory Animal Care formulated by the National Society for Medical Research and to NIH guidelines (Guide for the Care and Use of Laboratory Animals. National Academies Press. 1996.), and all in vivo procedures were performed by UK Home Office Personal License holders. All animal studies were performed with the approval of the ethics committee of the Queen Mary University of London and the UK Home Office (project license PPL70/7254).

\section{Author contributions}

MS, Yasunori Shintani, and KS conceived and designed the research studies. MS and Yusuke Shintani conducted most of the experiments and acquired data, with professional support from Yasunori Shintani, HI, RS, AY, HA, KY, and KS. All authors participated in the analysis and interpretation of the data. KS primarily wrote and edited the manuscript, with input from all other authors.

\section{Acknowledgments}

This project was funded by K. Suzuki's discretionary fund, a Queen Mary Innovation Proof of Concept Grant, and the NIH Research-funded Barts Cardiovascular Biomedical Research Unit. M. Shiraishi was supported by a Fukuda Memorial Foundation 
Fellowship and a Uehara Memorial Foundation Research Fellowship. Trib1/-/ mice were a gift of S. Akira and T. Sato (Immunology Frontier Research Center, Osaka University, Osaka, Japan) under a material transfer agreement.
Address correspondence to: Ken Suzuki, William Harvey Research Institute, Barts and The London School of Medicine and Dentistry, Charterhouse Square, London, EC1M 6BQ, United Kingdom. Phone: 44.20.7882.8233; E-mail:ken.suzuki@qmul.ac.uk.
1. van den Borne SW, Diez J, Blankesteijn WM, Verjans J, Hofstra L, Narula J. Myocardial remodeling after infarction: the role of myofibroblasts. Nat Rev Cardiol. 2010;7(1):30-37.

2. Shinde AV, Frangogiannis NG. Fibroblasts in myocardial infarction: a role in inflammation and repair. J Mol Cell Cardiol. 2014;70:74-82.

3. Porrello ER, et al. Transient regenerative potential of the neonatal mouse heart. Science. 2011;331(6020):1078-1080.

4. Gordon S, Martinez FO. Alternative activation of macrophages: mechanism and functions. Immunity. 2010;32(5):593-604.

5. Wang G, et al. HDAC inhibition prevents white matter injury by modulating microglia/ macrophage polarization through the GSK3 $\beta$ / PTEN/Akt axis. Proc Natl Acad Sci U S A. 2015;112(9):2853-2858.

6. Lee $S$, et al. Distinct macrophage phenotypes contribute to kidney injury and repair. J Am Soc Nephrol. 2011;22(2):317-326.

7. Heidt $\mathrm{T}$, et al. Differential contribution of monocytes to heart macrophages in steadystate and after myocardial infarction. Circ Res. 2014;115(2):284-295.

8. Epelman S, et al. Embryonic and adult-derived resident cardiac macrophages are maintained through distinct mechanisms at steady state and during inflammation. Immunity. 2014;40(1):91-104.

9. Nahrendorf $\mathrm{M}$, et al. The healing myocardium sequentially mobilizes two monocyte subsets with divergent and complementary functions. JExp Med. 2007;204(12):3037-3047.

10. Yang $M$, et al. Serum-glucocorticoid regulated kinase 1 regulates alternatively activated macrophage polarization contributing to angiotensin II-induced inflammation and cardiac fibrosis. Arterioscler Thromb Vasc Biol. 2012;32(7):1675-1686.

11. Lörchner $\mathrm{H}$, et al. Myocardial healing requires Reg3 $\beta$-dependent accumulation of macrophages in the ischemic heart. Nat Med. 2015;21(4):353-362.

12. Ben-Mordechai T, et al. Macrophage subpopulations are essential for infarct repair with and without stem cell therapy. J Am Coll Cardiol. 2013;62(20):1890-1901.

13. Ma Y, et al. Matrix metalloproteinase- 28 deletion exacerbates cardiac dysfunction and rupture after myocardial infarction in mice by inhibiting M2 macrophage activation. Circ Res. 2013;112(4):675-688.

14. van Rooijen N, Hendrikx E. Liposomes for specific depletion of macrophages from organs and tissues. Methods Mol Biol. 2010;605:189-203.

15. Fujiu K, Wang J, Nagai R. Cardioprotective function of cardiac macrophages. Cardiovasc Res. 2014;102(2):232-239.

16. Satoh T, et al. Critical role of Trib1 in differentiation of tissue-resident M2-like macrophages.
Nature. 2013;495(7442):524-528.

17. Nichols M, et al. European Cardiovascular Disease Statistics 2012. Brussels, Belgium: European Heart Network, Brussels, European Society of Cardiology, Sophia Antipolis; 2012. https://www. escardio.org/static_file/Escardio/Press-media/ press-releases/2013/EU-cardiovascular-disease-statistics-2012.pdf. Accessed April 4, 2016.

18. Harel-Adar T, Ben Mordechai T, Amsalem Y, Feinberg MS, Leor J, Cohen S. Modulation of cardiac macrophages by phosphatidylserine-presenting liposomes improves infarct repair. Proc Natl Acad Sci U S A. 2011;108(5):1827-1832.

19. Weirather J, et al. Foxp $3^{+} \mathrm{CD}^{+} \mathrm{T}$ cells improve healing after myocardial infarction by modulating monocyte/macrophage differentiation. Circ Res. 2014;115(1):55-67.

20. Leor J, et al. Ex vivo activated human macrophages improve healing, remodeling, and function of the infarcted heart. Circulation. 2006;114(1 suppl):I94-I100.

21. Torre-Amione G, et al. Advanced Chronic Heart Failure Clinical Assessment of Immune Modulation Therapy Investigators. Results of a non-specific immunomodulation therapy in chronic heart failure (ACCLAIM trial): a placebo-controlled randomised trial. Lancet. 2008;371(9608):228-236.

22. Harel-Adar T, Ben Mordechai T, Amsalem Y, Feinberg MS, Leor J, Cohen S. Modulation of cardiac macrophages by phosphatidylserine-presenting liposomes improves infarct repair. Proc Natl Acad Sci U S A. 2011;108(5):1827-1832.

23. Jenkins SJ, et al. Local macrophage proliferation, rather than recruitment from the blood, is a signature of TH2 inflammation. Science. 2011;332(6035):1284-1288.

24. Rückerl D, et al. Induction of IL-4R $\alpha$-dependent microRNAs identifies PI3K/Akt signaling as essential for IL-4-driven murine macrophage proliferation in vivo. Blood. 2012;120(11):2307-2316.

25. Martinez FO, et al. Genetic programs expressed in resting and IL-4 alternatively activated mouse and human macrophages: similarities and differences. Blood. 2013;121(9):e57-e69.

26. Sica A, Mantovani A. Macrophage plasticity and polarization: in vivo veritas. JClin Invest. 2012;122(3):787-795.

27. Terrazas LI, Montero D, Terrazas CA, Reyes JL, Rodríguez-Sosa M. Role of the programmed death-1 pathway in the suppressive activity of alternatively activated macrophages in experimental cysticercosis. Int J Parasitol. 2005;35(13):1349-1358.

28. Kurokawa J, et al. Apoptosis inhibitor of macrophage (AIM) is required for obesity-associated recruitment of inflammatory macrophages into adipose tissue. Proc Natl Acad Sci U S A. 2011;108(29):12072-12077.

29. Guo H, Jin D, Chen X. Lipocalin 2 is a regulator of macrophage polarization and $\mathrm{NF}-\kappa \mathrm{B} /$
STAT3 pathway activation. Mol Endocrinol. 2014;28(10):1616-1628.

30. Cucak H, Nielsen Fink L, Højgaard Pedersen M, Rosendahl A. Enalapril treatment increases T cell number and promotes polarization towards M1-like macrophages locally in diabetic nephropathy. Int Immunopharmacol. 2015;25(1):30-42.

31. Yan $\mathrm{X}$, et al. Temporal dynamics of cardiac immune cell accumulation following acute myocardial infarction. J Mol Cell Cardiol. 2013;62:24-35.

32. Krishnamurthy P, Rajasingh J, Lambers E, Qin G, Losordo DW, Kishore R. IL-10 inhibits inflammation and attenuates left ventricular remodeling after myocardial infarction via activation of STAT3 and suppression of HuR. Circ Res. 2009;104(2):e9-18.

33. Suzuki K, et al. Overexpression of interleukin-1 receptor antagonist provides cardioprotection against ischemia-reperfusion injury associated with reduction in apoptosis. Circulation. 2001;104(12 suppl 1):I308-I3I3.

34. Giacca M, Zacchigna S. VEGF gene therapy: therapeutic angiogenesis in the clinic and beyond. Gene Ther. 2012;19(6):622-629.

35. Aden N, et al. Epithelial cells promote fibroblast activation via IL-1alpha in systemic sclerosis. J Invest Dermatol. 2010;130(9):2191-2200.

36. Suwara MI, et al. IL-1 $\alpha$ released from damaged epithelial cells is sufficient and essential to trigger inflammatory responses in human lung fibroblasts. Mucosal Immunol. 2014;7(3):684-693.

37. Trueblood NA, et al. Exaggerated left ventricular dilation and reduced collagen deposition after myocardial infarction in mice lacking osteopontin. Circ Res. 2001;88(10):1080-1087.

38. Singh M, Foster CR, Dalal S, Singh K. Osteopontin: role in extracellular matrix deposition and myocardial remodeling post-MI. J Mol Cell Cardiol. 2010;48(3):538-543.

39. Suzuki T, et al. Pulmonary macrophage transplantation therapy. Nature. 2014;514(7523):450-454.

40. Saggini A, et al. Allergic inflammation: role of cytokines with special emphasis on IL-4. Int J Immunopathol Pharmacol. 2011;24(2):305-311.

41. Kleemann R, Zadelaar S, Kooistra T. Cytokines and atherosclerosis: a comprehensive review of studies in mice. Cardiovasc Res. 2008;79(3):360-376.

42. Braga TT, et al. MyD88 signaling pathway is involved in renal fibrosis by favoring a TH2 immune response and activating alternative M2 macrophages. Mol Med. 2012;18:1231-1239.

43. Prendiville J, et al. Recombinant human interleukin-4 (rhu IL-4) administered by the intravenous and subcutaneous routes in patients with advanced cancer - a phase I toxicity study and pharmacokinetic analysis. Eur J Cancer. 1993;29A(12):1700-1707.

44. Wiernik PH, et al. Phase II study of interleukin-4 in indolent B-cell non-Hodgkin lymphoma and B-cell chronic lymphocytic leukemia: a study 


\section{RESEARCH ARTICLE}

of the Eastern Cooperative Oncology Group (E5Y92). J Immunother. 2010;33(9):1006-1009.

45. Tano N, et al. Epicardial placement of mesenchymal stromal cell-sheets for the treatment of ischemic cardiomyopathy; in vivo proof-of-concept study. Mol Ther. 2014;22(10):1864-1871.

46. Shintani Y, et al. TLR9 mediates cellular protec- tion by modulating energy metabolism in cardiomyocytes and neurons. Proc Natl Acad Sci U S A. 2013;110(13):5109-5114.

47. Zhang X, Goncalves R, Mosser DM. The isolation and characterization of murine macrophages.

Curr Protoc Immunol. 2008; Chapter 14:Unit 14.1.

48. Narita T, et al. The use of cell-sheet technique eliminates arrhythmogenicity of skeletal myoblast-based therapy to the heart with enhanced therapeutic effects. Int J Cardiol. 2013;168(1):261-269.

49. Leicht M, Greipel N, Zimmer H. Comitogenic effect of catecholamines on rat cardiac fibroblasts in culture. Cardiovasc Res. 2000;48(2):274-284. 